Heinrich ZABEHLICKY

\title{
ZUM ABSCHLUSS DER GRABUNGEN IM HAUPTGEBÄUDE DER VILLA VON BRUCKNEUDORF
}

Dieser Bericht kann unmittelbar nach dem Abschluß der letzten Grabungskampagne nur eine Rohbilanz der Arbeit der letzten zehn Jahre darstellen. Manches wird in der endgültigen Publikation zu präzisieren oder auch zu korrigieren sein. Dennoch soll eine vorläufige Zusammenschau des Erreichten vorgelegt werden.

\section{Voraussetzungen}

An dem großen Baukomplex hatten schon dreimal Grabungen stattgefunden: Eine Testgrabung von A. Seracsin 1931, die weiträumige Grabung des Platzes einschließlich der Nebengebäude und der Umfassungsmauern durch B. Saria in den Jahren 1949-1955 und die Hebung der Mosaiken durch G. Langmann 1975 und 1976, an die sich bis 1987 Untersuchungen im Hauptgebäude und im Bad angeschlossen haben. 1989 wurden vor der Errichtung der Masten für die 380-KV-Stromleitung die Standorte dieser Masten untersucht, von denen einer im ummauerten Areal der Villa, aber nicht im Bereich des Hauptgebäudes lag. Eine Darstellung der Grabungsgeschichte bis 1987, soweit sie nachzuvollziehen ist, bleibt der endgültigen Publikation vorbehalten.

Die Dokumentationen der früheren Grabungen entsprachen dem Standard der Zeit, in der sie erarbeitet worden waren. Ein Bericht von A. Seracsin liegt in mehreren gleichlautenden Exemplaren vor ${ }^{1}$, wurde aber in den $»$ Fundberichten aus Österreich « sehr verkürzt wiedergegeben $^{2}$. Von B. Saria liegt das maschinschriftliche Tagebuch im Archiv des Österreichischen Archäologischen Instituts (ÖAI) vor, im Planarchiv desselben auch die von J. Hierath erstellten Pläne sowie einige Planunterlagen. Erschienen sind jährliche Vorberichte ${ }^{3}$ und eine zusammenfassende Darstellung ${ }^{4}$. Aus der Grabungsperiode unter der Leitung von G. Langmann verwahrt das Institutsarchiv zwei Versionen eines Gesamtplanes, handschriftliche und für einen Teil der Kampagnen auch maschinschriftliche Tagebücher, Zeichnungen von Profilen, Maueransichten, Baudetails (Schwellen) und Ziegelstempeln, Schwarzweißphotos und Diapositive. Die Schwarz-

\footnotetext{
Im Burgenländischen Landesmuseum und am Österreichischen Archäologischen Institut.

2 A. Seracsin, Parndorfer Heide, FÖ 1, 1930-34, 94.

3 G. Pascher, Bericht über die Versuchsgrabung Parndorf (Oktober 1949), BHBl 12, 1950, 49-54; B. Saria, Der Mosaikenfund von Parndorf zwischen Parndorf und Bruckneudorf, BHB1 13, 1951, 49-65 (In dem mir vorliegenden Exemplar so gedruckt: Handschriftlich ist »von Parndorf« zu »vom Heidehof« ausgebessert. Offensichtlich war der ursprünglich von Saria geplante Titel »Der Mosaikenfund von Parndorf«. Erst in den Korrekturen wurde hinzugefügt, daß der Fundort zwischen den beiden Orten liegt, doch wurde »von Parndorf« nicht mehr durch »vom Heidehof« ersetzt. Saria kam wohl immer vom näher gelegenen Parndorf und hat erst in seinem zusammenfassenden Bericht auf die Lage im Gemeindegebiet von Bruckneudorf hingewiesen, aber an der Bezeichnung Parndorf festgehalten. Es ist auch heute noch schwierig, in Ungarn über »Bruckneudorf« ohne den Zusatz »Parndorf [Pándorfalu] « zu sprechen. Unter diesem Namen ist der Fundort seit E. B. Thomas, Römische Villen in Pannonien [1964] 177-192 sehr bekannt.); ders., Zweiter vorläufiger Bericht über die Grabungen nächst dem Heidehof zwischen Parndorf und Bruckneudorf, BHB1 14, 1952, 97-102.

4 B. Saria, Der römische Herrensitz bei Parndorf und seine Deutung, in: Festschrift A. A. Barb, WAB 35 (1966) 252-271.
} 


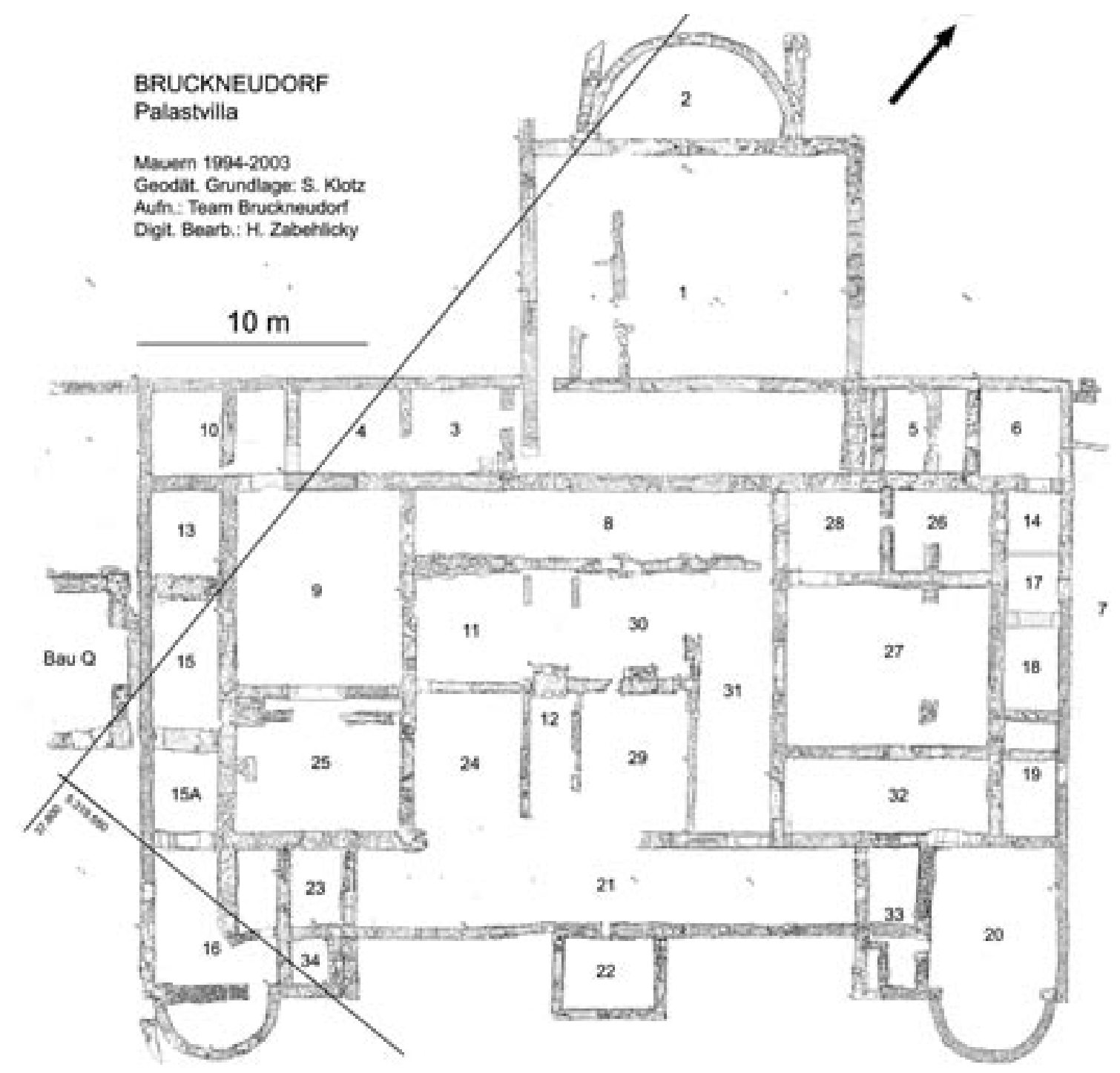

1 Gesamtplan der Mauern nach den Aufnahmen 1994-2003 mit Raumnummern

weißphotos waren nach Jahren geordnet, für die Hauptmasse der Diapositive mußte eine Zuordnung nach dem Bildinhalt versucht werden. Über die ersten Jahre dieser Grabungsperiode liegt ein Bericht vor ${ }^{5}$.

Gegeben war nach den Gesamtplänen der Grundriß der Periode mit Ziegeldurchschuß und Mosaikböden sowie eine seit B. Saria festgelegte Numerierung der Räume. Etliche Informationen waren den publizierten Vorberichten und Zusammenfassungen zu entnehmen ${ }^{6}$. Die Lage der Sondagen der früheren Grabungsperioden war aber nur gelegentlich in den Plänen eingetragen, sie mußte also nach dem Befund selbst und nach den Angaben in den Tagebüchern bzw. den nicht allzu reichlich vorhandenen Profilzeichnungen zugeordnet werden.

Die Kenntnis der Baugeschichte zu Beginn der Untersuchungen durch den Verfasser kann mit folgenden Zitaten umrissen werden:

5 G. Langmann, Bericht über die Grabungskampagnen 1975-1978 in Bruckneudorf, Bezirk Neusiedl am See, Burgenland, BHB1 41, 1979, 66-87 und 100-114.

6 Neben den soeben zitierten Zusammenfassungen sind Berichte in PAR, FÖ und ÖJh erschienen. Bis 1999 sind sie zusammengestellt bei: H. Zabehlicky, Fünf Grabungsjahre im Hauptgebäude der Villa von Bruckneudorf/Burgenland, 1994-1998, PAR 49, 1999, 13 Anm. 1 und 2. 
G. Langmann hatte zwei Perioden definiert, wobei nur für Periode II eine absolutchronologische Angabe gemacht werden konnte: $» \ldots$ diese fällt demnach in das 3. Viertel des 4. Jahrhundert n. Chr., also in die Zeit bald nach der Jahrhundertmitte ...«. Für Periode I gibt er mangels datierender Funde an: »Rein hypothetisch möchte man I in die Zeit um 300 n. Chr. ansetzen. « ${ }^{7}$ Dazu kommt ein Vorgängerbau in Rutenputz- und Fachwerktechnik, den G. Langmann folgendermaßen umschreibt: »Es scheint ziemlich unwahrscheinlich, daß dieser Vorläufer aus Holz und Mörtel schon den Grundriß von I und II vorweggenommen hat. « ${ }^{8}$ In ihrer von G. Langmann betreuten Diplomarbeit folgte I. Benda im wesentlichen dieser Aufgliederung, wobei sie aber den Rutenputzbau schon als eigene Periode bestimmte: Periode I: Rutenputz, Periode II: Steinbau mit ähnlichem Grundriß wie die folgende Periode III: Steinbau mit Mosaikausstattung und Zubau bald nach 350 n. Chr. ${ }^{9}$. Sie erkannte auch den Ziegeldurchschuß als charakteristisch für ihre Periode III ${ }^{10}$. Eine Überprüfung durch Kontrollgrabungen war ihr nicht möglich.

Diese Angaben beruhten auf kleineren Sondagen, die unter die Estrichböden bzw. Mosaikunterbauten abgetieft worden waren. In der Dokumentation fanden sich als Notizen auf Zeichnungen immer wieder Hinweise auf mehrfache Fundamentierungen, doch konnten diese kaum zugeordnet werden. Die aus der Dokumentation erkennbare Situation war Anlaß, doch größere Teile der Räume auszugraben, um Zusammenhänge besser zu erfassen. Trotzdem sollten aber Mauern und auch Heizkanäle, soweit sie noch vorhanden waren, möglichst nicht abgetragen werden.

\section{Die Grabungen seit 1994}

Die 'neuen' Grabungen begannen am 7. Juni 1994. Geplant war damals, über dem gegebenen Zustand einen Schutzbau zu errichten. Dafür sollten allerdings auch Mittel eingesetzt werden, die dem ÖAI zur Verfügung standen und besser für Forschungsaufgaben verwendet werden konnten. Der damalige Direktor des Instituts, D. Knibbe, hat daher mit einer internen Kommission $^{11}$ eine Besichtigung des Platzes vorgenommen, um die Situation gemeinsam zu beurteilen. Man kam zu dem Entschluß, daß vor der Errichtung eines Schutzbaus die vollständige Erforschung durchgeführt werden sollte. Mit dieser Aufgabe wurde der Verfasser betraut.

Die Zurückziehung des Beitrages zum Schutzbau durch das ÖAI rief natürlich bei dem Land Burgenland als Grundeigentümer und bei der Gemeinde Bruckneudorf verständlichen Unmut hervor; beide hatten erwartet, bald über ein repräsentatives antikes Denkmal zu verfügen und dieses auch touristisch vermarkten zu können. In dieser Situation war es geboten, die Grabungsarbeiten rasch zu beginnen, um die Bereitschaft des Instituts zu demonstrieren, dieses Projekt nicht über das für die Forschung notwendige Maß hinaus zu verzögern. Nach knapper Vorbereitungszeit wurde die Grabung am 15. Juli 1994 begonnen.

Unter den beschriebenen Voraussetzungen des Forschungs- und Wissenstandes war es am sinnvollsten, sowohl den Grabungsvorgang als auch die Ordnung der Dokumentation an der gegebenen Raumeinteilung auszurichten (Abb. 1). In den unten folgenden Beschreibungen der den einzelnen Perioden zugeordneten Befunde wird daher immer wieder auf die Raumnummern Bezug genommen, auch wenn viele Räume in einigen Perioden noch nicht vorhanden waren. Für die Vermessung wurde im Jahr 1995 von St. Klotz (ÖAI Wien) eine Anzahl von Punkten aufgenommen und später ergänzt; diese steht als 'Punktwolke' zur Verfügung. Die gescannten

7 Langmann (Anm. 5) 71.

8 Ebenda 72.

9 I. Benda, Der Gutshof von Bruckneudorf und seine Stellung innerhalb der Villenarchitektur der römischen Kaiserzeit (ungedr. Mag. Wien 1989) 75-79.

10 Ebenda 40.

1 Sie bestand aus D. Knibbe selbst, M. Kandler, P. Scherrer und H. Zabehlicky als Ausgräber im Inland, H. Thür als Bauforscherin und K. Herold als Restaurator. 


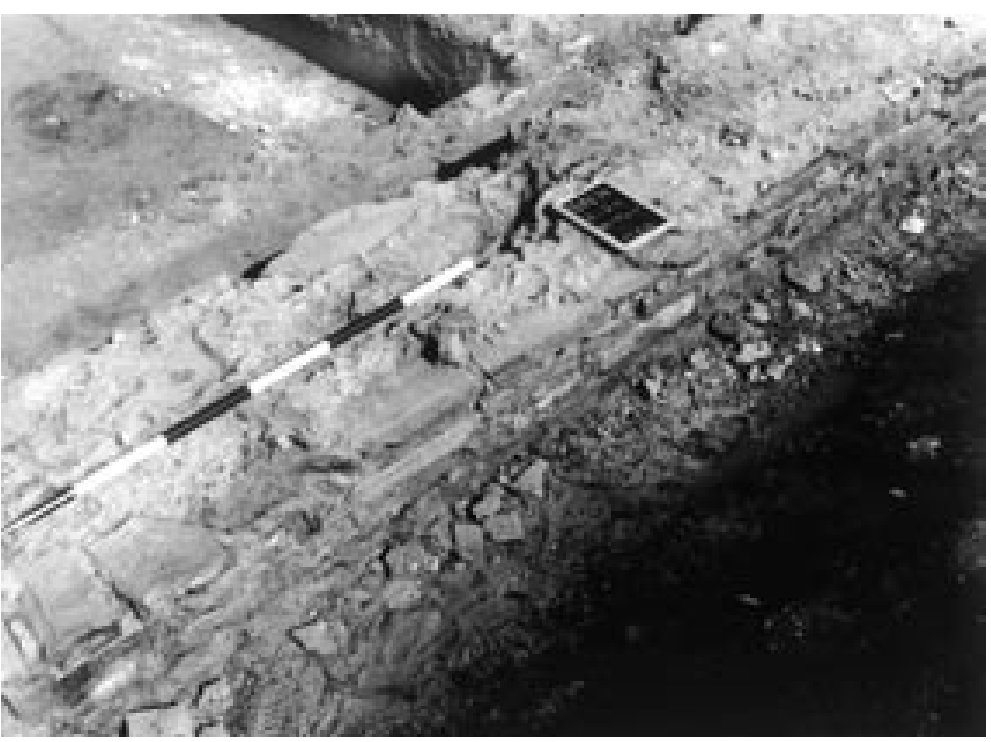

2 Ziegeldurchschuß der Westmauer von Raum 1
Zeichnungen konnten sodann problemlos zu einem Gesamtplan zusammengefügt werden ${ }^{12}$. Für die Höhenlage wurde als einheitlicher Ausgangspunkt die Schwelle zwischen den Räumen 9 und 25 gewählt, die nach der vorliegenden Vermessung 156,200 m über dem Mittelwasser der Adria bei Triest liegt. Grundrisse und Plana wurden im Maßstab 1:50 aufgenommen, Profile und Maueransichten im Maßstab 1:20. Als Grabungsnord wurde die Seite des Baus festgelegt, an der der große Raum 1 mit der

Apsis 2 liegt. Soweit in den früheren Berichten Himmelsrichtungen angegeben sind, bezeichnen sie meist, aber nicht durchgehend, die Flanke mit den Räumen 6, 14, 17, 18, 19 und 20 als Nord. Tatsächlich liegt die Villa fast diagonal in bezug auf die Himmelsrichtungen, doch ist die Abweichung bei der hier gewählten Vereinfachung kleiner $\left(39^{\circ}\right)$ als bei der in den früheren Berichten gewählten $\left(51^{\circ}\right)$.

Die Kampagnen dauerten außer in den ersten beiden Jahren (1994: 6 Wochen; 1995: 11 Wochen) durchweg 8-9 Wochen und wurden in den Monaten Juli/August durchgeführt. Zunächst wurde in den Räumen 1 und 21 mit der Arbeit begonnen und ein Streifen am Westrand des Baus untersucht, um einen Überblick über die allgemeine Stratigraphie außerhalb des Bauwerks zu gewinnen. Auch Raum 1 war über eine lange Zeit der Baugeschichte nicht verbaut, damit konnte hier ebenfalls eine Fläche außerhalb des Gebäudes untersucht werden. Estriche und die darunterliegenden Bürsten mußten allerdings in vielen Räumen teilweise abgetragen werden. In allen Räumen, in denen noch eine originale Schichtfolge vorhanden war, wurden Riegel belassen, die eine spätere Kontrolle ermöglichen sollen. In abgeschlossenen Grabungsabschnitten wurden die tiefsten jeweils erreichten Niveaus markiert und geschützt, indem auf Erdplana gelbroter Schotter (Dicke ca. 0,05 m) aufgebracht wurde, Estriche und Mauern wurden durch das Auflegen von Bauvlies zusätzlich geschützt.

In dieser Weise konnten die Grabungsarbeiten seit 1997 jeweils in mehreren Räumen abgeschlossen werden ${ }^{13}$.

\section{Baugeschichte}

Nach dem oben zusammengefaßten Stand der Kenntnis der Baugeschichte vor 1994, die vor allem die späteste Bauperiode erfaßte, mußte diese als Ausgangspunkt dienen ${ }^{14}$. Die Periode war durch den Mosaikschmuck ausgezeichnet, als bautechnisches Kriterium kann die Verwendung

12 Verwendet wurde das handelsübliche Graphikprogramm Adobe Photoshop 6.0.

13 1997: Raum 34; 1998: Räume 21, 22, 23, 24, 29; 1999: Räume 1, 13, 25, 27, 33; 2000: Räume 2, 15, 15A, 16, 19, 20, 32; 2001: Räume 12, 14, 17, 18, 30; 2002: Räume 5, 6, 26, 28, 31; 2003: Räume 3, 4, 8, 9, 10, 11.

14 Die Schwierigkeiten derartiger Nachgrabungen auf bereits mehrfach ausgegrabenen Plätzen sind allen bekannt, die mit solchen Aufgaben betraut sind. Unter Einbeziehung und Wertung der vorhandenen Dokumentation hofft der Verf. dennoch, zu einem schlüssigen Ergebnis zu kommen. 
von Ziegeldurchschuß gelten. Besonders deutlich mit bis zu fünf Ziegellagen übereinander war das an der Westmauer des Saales Raum $1 \mathrm{zu}$ erkennen (Abb. 2): Soweit zu sehen, waren große Bruchstükke von tegulae verwendet worden.

Ein weiteres Charakteristikum konnte an zahlreichen anderen Mauern festgestellt werden. Es ist dies ein markanter Fugenstrich in den Mörtellagen zwischen den Steinen (Abb. 3). Viele dieser Mauern wurden in der letzten Phase weiterverwendet. Bei einigen dieser Fugenstrichmauern zeigte sich über ihrer untersten Fundamentschar eine schwache

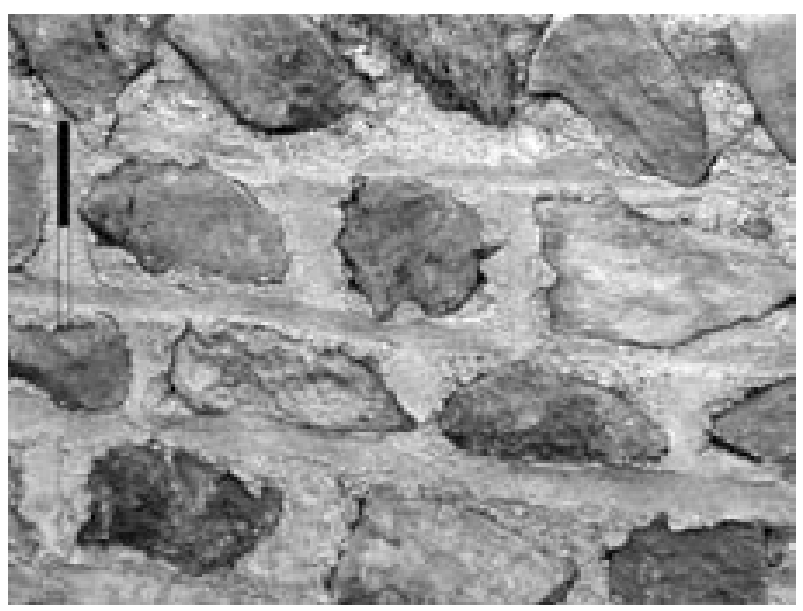

3 Fugenstrichmauerwerk in Raum 27 Lage von Lehmbrocken, die mit Humus vermischt war, in einer Stärke von meist 0,04 m, gelegentlich fehlte die Lage auf kürzeren Strekken, gelegentlich war sie bis $0,08 \mathrm{~m}$ dick. Soweit die ursprünglichen Schichten noch vorhanden waren, fand sich diese Lage in der Tiefe des antiken Humus, noch über dem gewachsenen sandigen Aulehm. Es handelt sich damit wohl um ältere Fundamente, die meist nur aus einer Steinschar bestanden. Der Aufbau darüber ist als Holzfachwerkkonstruktion anzunehmen.

An mehreren Stellen wurden große Pfostengruben angetroffen. Sie greifen tief in den gewachsenen Boden ein und messen ca. 0,55 × 0,70 m. Die Öffnung größerer Flächen hat es erlaubt festzustellen, daß das System dieser Pfostengruben einer anderen Orientierung folgt als die Mauern.

Damit war der Ansatz für vier bautechnisch grundsätzlich verschiedene Bauperioden gegeben, der bereits vorgelegt wurde ${ }^{15}$ und später noch ergänzt werden konnte ${ }^{16}$. Die folgende Vorstellung des Befundes ist nach diesen Perioden eingeteilt, für die auch, soweit möglich, ein Datierungsvorschlag gemacht wird. Angeschlossen ist jeweils eine vorläufige Beurteilung mit ersten Hinweisen auf vergleichbare Befunde.

\section{Die Holzbauperiode}

Für die Holzbauperiode haben sich weitere Befunde ergeben (Abb. 4). In der Darstellung sind alle 'Verfärbungen' eingetragen, diejenigen, die zu dieser Periode gehören, aber schwarz ausgeführt. Grau angelegt sind die Flächen, wo Mauern, Kanäle und gut erhaltene Estrichböden es nicht erlaubt haben, bis auf den gewachsenen Boden zu graben.

Abgesehen von wenigen Gräbchen wurden zumeist große Pfostengruben gefunden, in denen häufig auch noch die Pfostenspur zu erkennen war. Deutlich ist die andere Orientierung gegenüber den späteren Perioden. Nachdem sich in Raum 1 eine Reihe fünf derartiger Pfosten in einigermaßen regelmäßigen Abständen gezeigt hatte, konnte ein regelmäßiges System angenommen werden. Das gezielte Öffnen von Böden hat dann auch erlaubt, den Grundriß zu ergänzen. Unter dem Südteil des späteren Gebäudes (Räume 24, 12 und 29) zeigten sich außer Pfostengruben auch einige Gräbchen im gewachsenen Boden.

Ein Datierungsvorschlag stützt sich zunächst auf die ältesten Funde, die im gesamten Bereich zutage gekommen sind. Wegen der späteren Baumaßnahmen und der dadurch bewirkten Materialverlagerungen liegen sie keineswegs nur in den Schichten und Verfüllungen der Holzbau-

\footnotetext{
15 H. Zabehlicky, Bruckneudorf, ÖJh 64, 1995, Beibl. Grabungen 1994, 54-56 Abb. 1; Ch. Öllerer - H. Zabehlicky, Bruckneudorf, FÖ 36, 1997, 822 f.; Zabehlicky (Anm. 6) 7-14 Abb. 1.

16 Ch. Öllerer - H. Zabehlicky, Bruckneudorf, FÖ 40, 2001, 618-620 mit Abb. 394.
} 


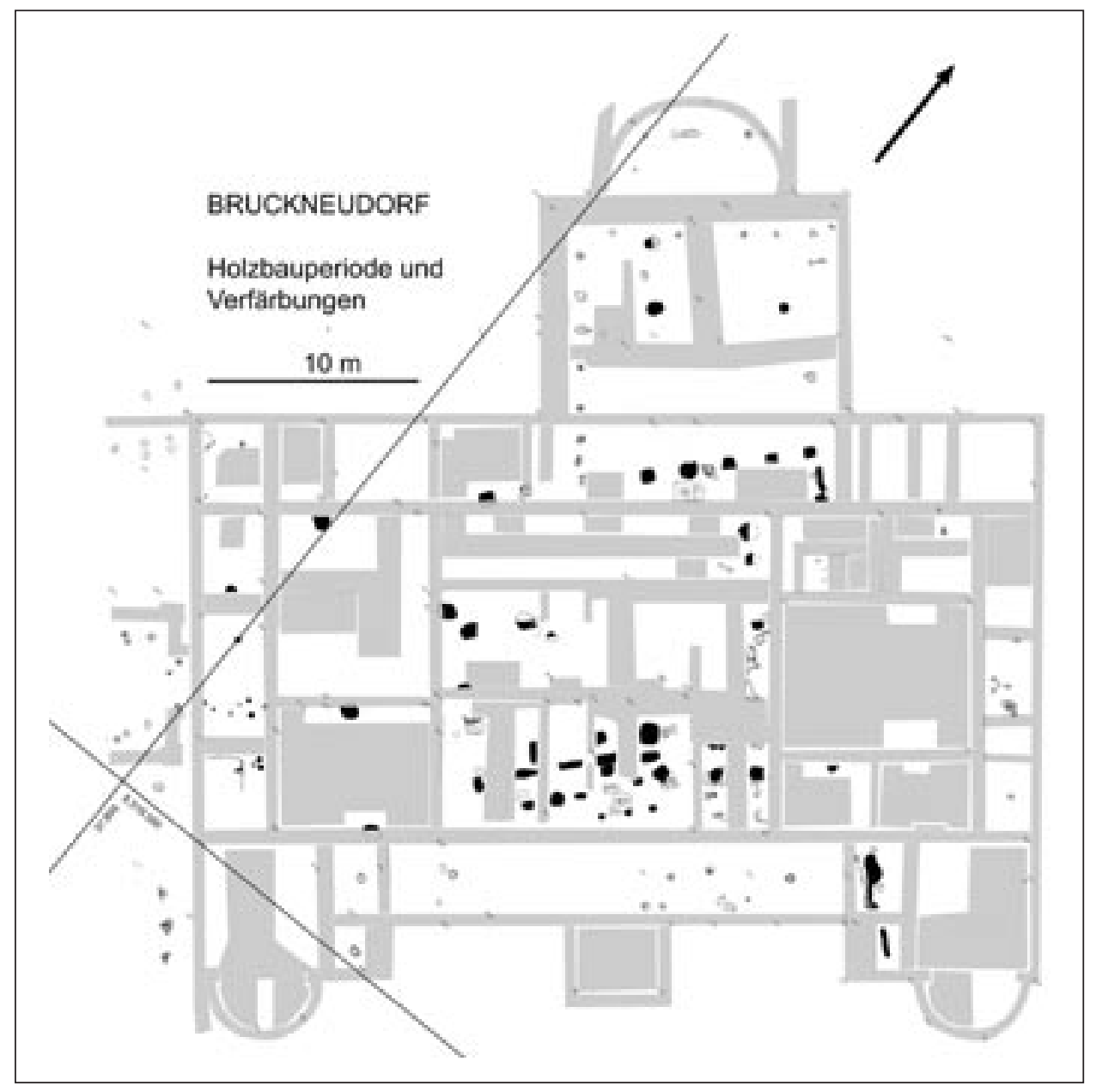

4 Plan der Holzbauperiode

periode. Es ergibt sich ein erster Schwerpunkt in flavischer Zeit. Einige Stücke stammen aber aus klarer Fundlage. In dem Gräbchen in Raum 12 lag ein Denar des Galba und in der Verfüllung der Pfostengrube in Raum 9 ein winziges Bruchstück von Terra Sigillata, das aber als TardoPadana bestimmt werden kann, und in einer Pfostengrube in Raum 29 ein gestempelter Schalenboden Drag. 27 des Vitalis (ca. 65-80 n. Chr.) ${ }^{17}$.

Auch der jetzt vorliegende Grundriß ist bruchstückhaft und läßt noch einen weiten Spielraum der Interpretation zu. Ein erster Vorschlag zieht die Pfostenreihe unter Raum 1 heran, die nach Westen bis zu dem einzelnen Pfosten unter der Nordmauer von Raum 9 verlängert werden kann. Parallel zu dieser Pfostenreihe verläuft unter dem Südteil der späteren Steinbauten ein Gräbchen, vor dem, d. h. südlich dessen nochmals Pfosten in einer Reihe stehen, deren westlichster unter der Südmauer von Raum 25 erfaßt wurde. Die östliche Begrenzung wäre mit dem Nord-Süd verlaufenden Gräbchen in Raum 1 anzunehmen, ihr größter Teil war unter den Böden der Räume 28 und 27 nicht mehr sichtbar. Mit aller Vorsicht kann ein Rechteckhaus vorgeschlagen werden, das wenigstens an der Südseite Pfostenstellungen in Form eines Laubenganges hatte; die zusätzlichen Pfosten vor der Mitte der Südseite könnten einen Eingangsbereich markieren. An der Westseite lag vielleicht eine 'luftigere' Konstruktion vor, die mit weniger Pfosten auskam. Die

17 M. Polak, South Gaulish terra sigillata with potters' stamps from Vechten, 9. Suppl. Rei Cretariae Romanae Fautorum Acta (2000) 356 Taf. 25, V79. Die Aufnahme und erste Beurteilung des Fundmaterials verdanke ich meiner Frau S. Zabehlicky-Scheffenegger. 
Maße sind bei diesem Vorschlag, in den Achsen der Pfosten gemessen, 23,5 × $15 \mathrm{~m}$. Böden zu dieser Periode konnten nicht festgestellt werden.

Sicherlich ist diese Hypothese noch zu überprüfen, doch kann auf eine Parallele hingewiesen werden, die räumlich und chronologisch sehr nahe steht. In den canabae von Carnuntum wurde 1981/82 ein Rechteckhaus mit Umgängen an drei Seiten (Objekt 81 K) ergraben, dessen Außenmaße $15 \times 18$ m betragen $^{18}$. Die Maße weichen nicht allzusehr voneinander ab, allerdings liegt bei dem Haus in den canabae der Eingang mit einem kleinen Vordach an der Schmalseite. Diese frühe Verbauung kann in die Zeit ab der Mitte des 1. Jahrhunderts n. Chr. datiert werden.

Derselben Orientierung folgen ein Gräbchen im südöstlichen Teil der Villa (Raum 33), das nur auf einem kurzen Stück eindeutig faßbar war, und eine Reihe kleinerer Pfostengruben bzw. -löcher in den Räumen 13, 15 und 15A an der Westseite. Da kein Fundmaterial aus diesen Verfärbungen vorliegt, kann nur anhand der Orientierung eine vorsichtige Zuordnung zur Holzbauperiode getroffen werden. Drei größere Pfostengruben im Nordteil von Raum 1 sind ebenso tief eingegraben wie die zuvor beschriebenen. Mit Vorbehalt können sie ebenfalls noch zu dieser Periode gezählt werden. Die übrigen Verfärbungen, die dargestellt sind, entziehen sich vorläufig noch einer Deutung und einer Zuordnung zu einer der Perioden.

$\mathrm{Ob}$ dieser Bauzustand bis zum Beginn der folgenden Periode bestanden hat, oder ob ein Siedlungshiat vorliegt, kann bei dem derzeitigen Stand der Bearbeitung nicht sicher gesagt werden. In den Profilen zeigte sich mehrfach, daß die Gräbchen und Pfostengruben dieser Periode nicht direkt durch die Böden der folgenden Periode gekappt sind. Dazwischen lagen manchmal Humusschichten von ca. 0,10 m Dicke, manchmal ein Gemenge aus Humus mit teilweise verbranntem Lehm, das als Zerstörungsschutt der Holzbauperiode verstanden werden kann. Bei dem derzeitigen Stand der Bearbeitung kann ein Hiat nicht ausgeschlossen werden.

\section{Die Periode der 'älteren Fundamente'}

Wie erwähnt, konnten von der Periode der 'älteren Fundamente' nur an einigen Stellen die Fundamente erfaßt werden, die zumeist von späteren Mauern überbaut sind. Häufig handelt es sich nur mehr um eine Fundamentschar, über der Lehmbrocken mit Erde lagen, gelegentlich waren auch drei Scharen zu erkennen. In der vorläufigen Rekonstruktion des Planes (Abb. 5) sind die Stellen, an denen Fundamente unter späteren Mauern zu erkennen waren, durch Striche neben dem Mauerverlauf angegeben, soweit möglich auch die Abweichungen von den späteren Mauerfluchten.

Das aufgehende Mauerwerk dieser Periode war nirgends mehr erhalten. Nach dem Material, das verwendet wurde, um das Niveau für die folgende Periode zu erhöhen, kann es sich nur um Rutenputz gehandelt haben. Mörtelschutt, dessen Brocken deutliche Holzabdrücke zeigten, wurde in großen Mengen zur Anschüttung verwendet. Er zeigte eine gelblichweiße harte Oberfläche, das verwendete Holz waren zumeist Leisten mit rechteckigem Querschnitt von ca. 0,015 × 0,025 m. Daneben waren aber auch Lehmbrocken mit Rutenabdrücken und Verputzresten im Schutt reichlich vorhanden, ebenso Lehmziegel. Es kann bei dem gegenwärtigen Stand der Bearbeitung nicht gesagt werden, ob innerhalb dieser Periode mehrere Phasen vorliegen, oder ob manche Bauteile als Lehmfachwerk und andere als Mörtel-Rutenputz errichtet waren. Knapp nebeneinander liegende Mauern, wie an der Ostseite des Raumes 3 und an der Nordseite des Raumes 25, lassen eher vermuten, daß hier doch zwei Phasen vorliegen, deren Grundrisse in geringem Maße voneinander abweichen.

18 M. Kandler - H. Zabehlicky, Untersuchungen am Ostrand der Canabae Legionis von Carnuntum, in: Studien zu den Militärgrenzen Roms III, Forschungen und Berichte zur Vor- und Frühgeschichte in Baden-Württemberg 20 (1986) 341-349; M. Kandler, Die Lagerstadt von Carnuntum. Ergebnisse der Forschungsarbeiten des Jahres 1982 westlich des Kultbezirkes orientalischer Götter, MGFC I/83, 1983, 20-30 bes. 23 f. und Abb. 4 f. 


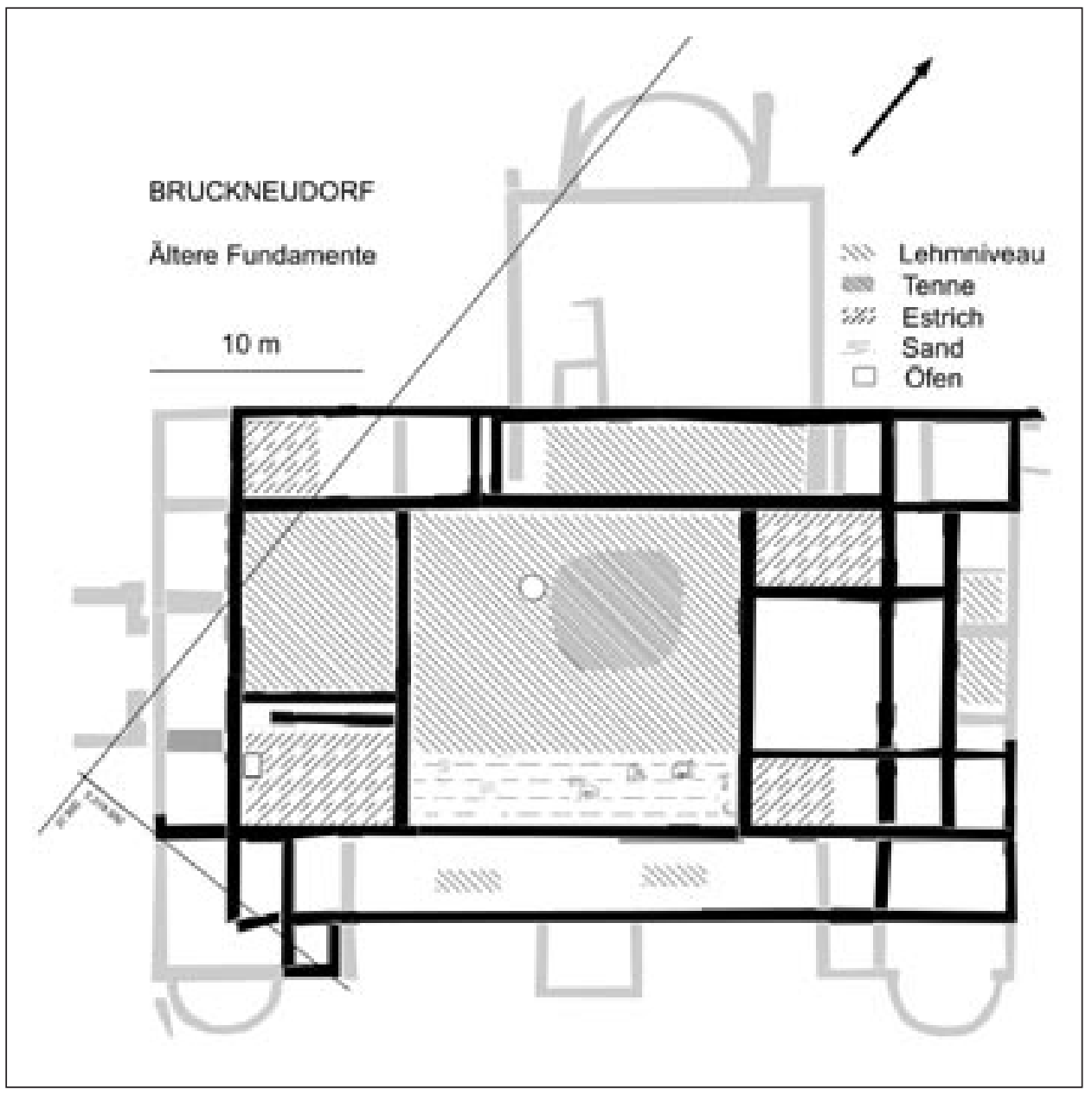

5 Plan der Periode der 'älteren Fundamente'

Großflächig konnte hingegen ein Lehmniveau festgestellt werden, dessen Ausdehnung auf dem Plan eingetragen ist. Es kommt auf der nicht überdachten zentralen Hoffläche vor, doch scheinen auch Innenräume einen solchen Boden besessen zu haben. In derselben Tiefe findet sich in einigen Innenräumen ein sehr harter Mörtelestrich, der stark mit Ziegelsplitt versetzt ist und eine deutlich rote Färbung aufweist: So ausgestattet waren ein Teil des späteren Raumes 10, Raum 28 mit einem Teil von 26, ein Teil von Raum 32 und Raum 25. In Raum 21 sind nur Reste des Lehmniveaus erhalten geblieben. Sowohl der Lehmboden als auch der Terrazzo fehlen verständlicherweise dort, wo spätere Hypokaustheizungen oder Praefurnia tiefer eingegriffen haben. Das ist in den Räumen 3, 19, 27 und dem Ostteil von Raum 32 der Fall. In Raum 21 sind nur Reste des Lehmniveaus erhalten geblieben.

Ein gemörtelter Aufbau auf dem Estrich an der Westwand des Raumes 25 kann gut als Unterbau eines Kuppelofens verstanden werden, der diesen Raum wohl als Küche bestimmt.

Im Hof zeigte sich noch, daß entlang seiner Südseite ein $3 \mathrm{~m}$ breiter Streifen nicht den üblichen Lehmboden aufwies, sondern mit sandigem Material etwa um 0,20 m höher planiert war (Abb. 6). Die Pfostengrube 31/10 an der Grenze von Sand und Lehm spricht dafür, daß hier ein Teil des Hofes mit einem Flugdach versehen war. Dasselbe Profil zeigen auch einen Pfosten (31/13) und ein Gräbchen (31/11) der Holzbauperiode, die aber deutlich von dem Lehmboden bzw. der sandigen Planierung überdeckt werden. Im Nordostteil des Hofes, auf dem Plan (Abb. 5) dichter schraffiert, zeigte der Lehmboden einen deutlich anderen Aufbau als an den übrigen Stellen, wo er geschnitten wurde. Sonst war es massiver gelber Lehm in einer Stärke von 0,04 
bis $0,08 \mathrm{~m}$, hier war er in zahlreichen Lagen gebändert, die durch feine dunklere Erdlagen getrennt waren (Abb. 7). Der Hof wurde also in diesem Teil regelmäßig, vielleicht jährlich, gereinigt und mit einer frischen Lehmschicht versehen. Ein so großer Bedarf an Reinlichkeit auf einem Wirtschaftshof läßt an eine Tenne denken. Die Untersuchung des Materials ergab einerseits eine große Anzahl von Phytolithen ${ }^{19}$

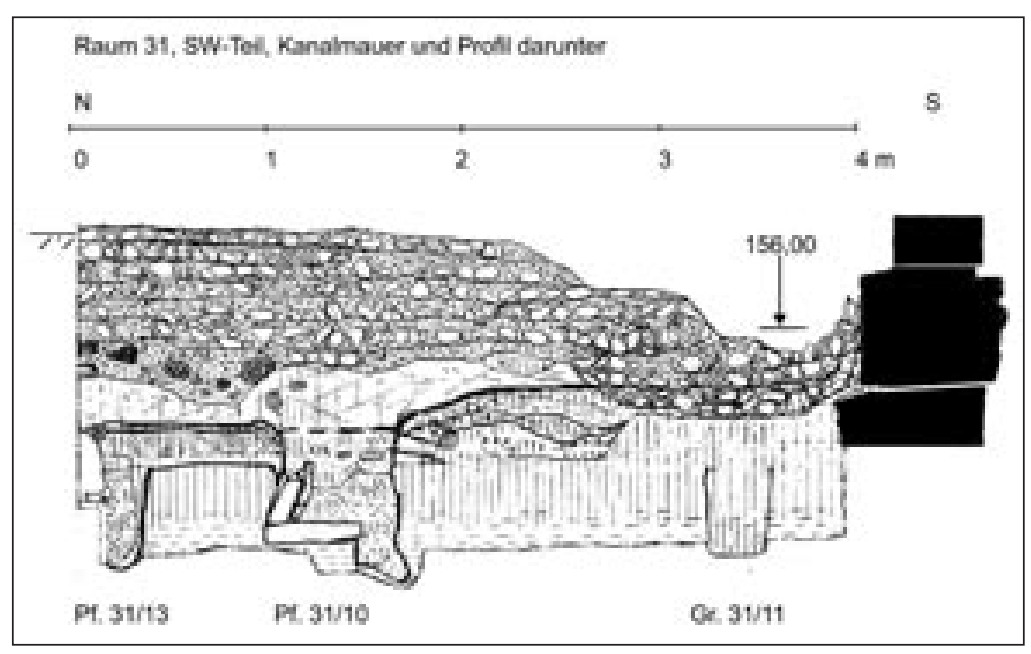

6 Profil mit Lehmboden und sandiger Planierung im Hof der Periode der 'älteren Fundamente' von Getreide, besonders von Weizen, und andererseits ein deutliches Überwiegen der Getreidepollen gegenüber anderen Pflanzen $^{20}$. Diese Ergebnisse lassen den Schluß zu, daß es sich hier um die Tenne der betreffenden Bauperiode der Villa handelt ${ }^{21}$.

Über den Zugang und die Aufschließung der einzelnen Räume können bei dem beschriebenen Erhaltungszustand kaum sichere Angaben gemacht werden. Ein Fundamentrest in leicht schräger Orientierung in der Südwestecke, unter Raum 16, könnte den Zugang markieren, der dann über den Gang Raum 21 zum zentralen Hof geführt hätte. Die Mauern, die diesen Zugang zu sperren scheinen, müssen nicht hochgezogen gewesen sein; die Mauer zwischen dem Gang und dem Hof ist zum größten Teil ausgebrochen.

Eine Datierung für diese Periode ist derzeit noch wenig abgesichert. Mit großer Wahrscheinlichkeit fällt die Errichtung in die erste Hälfte des 2. Jahrhunderts n. Chr. Wie oben erwähnt, ist ein Hiat nach der ersten Periode denkbar. Das Fundmaterial erlaubt nach einer ersten Durchsicht, für die Errichtung dieser Periode die trajanisch-hadrianische Zeit vorzuschlagen. Der Schutt dieser Periode, der zur Niveauerhöhung für die nächste verwendet worden ist, zeigte keine massiven Brandspuren oder Asche, so daß angenommen werden kann, daß diese Periode keiner gewaltsamen Zerstörung zum Opfer gefallen ist.

Die vorgeschlagene Grundrißlösung erscheint einigermaßen schlüssig. Das Gebäude schließt einen zentralen Hof ein, vier Räume sind mit Estrich versehen. Sie können als Wohnräume angesprochen werden. Abgesehen von der vorgeschlagenen Deutung des Raumes 25 als Küche können keine spezifischen Funktionen zugeordnet werden. Die Überdachung des Hofes entlang nur einer Seite erlaubt es nicht, von einer 'Peristylvilla' zu sprechen. Eine ausführliche

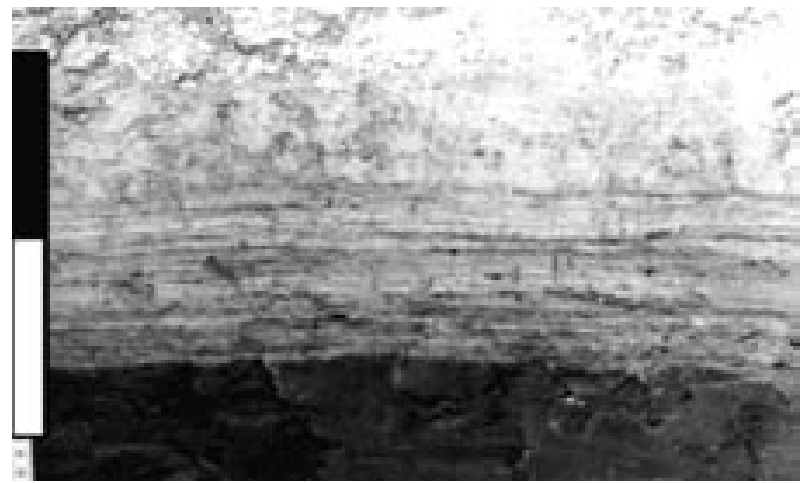

7 Schnitt durch den Lehmbelag der Tenne im Hof der Periode der 'älteren Fundamente'

19 Diese Bestimmungen führte U. Thanheiser (VIAS) durch.

20 Die Pollenanalyse wird I. Draxler (Geologische Bundesanstalt) verdankt.

${ }^{21}$ I. Draxler - U. Thanheiser - H. Zabehlicky, Eine Tenne aus der Villa von Bruckneudorf, in: Akten des 19. Internationalen Limeskongresses, Pécs 2003 (in Druck). 
Diskussion dieses Problems kann hier nicht vorgelegt werden, doch scheint die Anwendung der Polarität Peristylvilla gegenüber Porticusvilla (mit Eckrisaliten) für die Donauprovinzen insgesamt wenig zielführend. Das Begriffspaar wurde von K. M. Swoboda 1918 eingeführt, um große, vielfach palastartige Anlagen typologisch zu gliedern ${ }^{22}$, wobei auch der Gegensatz von 'griechischem' und 'römischem' Raumgefühl und Baudenken eine Rolle spielte. Beide Typen sind aber im 'repräsentativen' Wohnbau vertreten, und so sind auch Elemente beider Typen vielfach in das bescheidenere Bauen eingeflossen.

\section{Die Fugenstrichperiode}

Die Fugenstrichperiode ist die erste Bauperiode, von der aufgehendes Steinmauerwerk vorhanden ist. Wie erwähnt, folgt der Grundriß teilweise dem Bau, von dem nur mehr Fundamente faßbar waren, teilweise wird er aber auch stark geändert und erweitert (Abb. 8). Die 'älteren Fundamente' wurden mit zusätzlichen, oft in die etwas verbreiterten Fundamentgruben gegossenen Fundamenten überbaut und ausgeglichen. Darüber wurden die Mauern frei stehend aufgemauert, bei denen der Fugenstrich (Abb. 3) zur Anwendung kam. Erst als die Mauern bis zu dem angestrebten Niveau - ca. 0,70-0,80 m über dem früheren - aufgebaut waren, wurde das bereits erwähnte Abbruchmaterial des Rutenputzbaus eingefüllt. So erklärt sich, daß die Fugenstrichmauern (außer in Hypokausträumen) nicht verputzt sind und für sie keine Baugruben festgestellt werden konnten. Die Räume 3 und 27 wurden gemeinsam mit dem Ostteil von Raum 32 mit Hypokaustheizungen versehen. Die Stelle des Praefurniums für Raum 3 ist durch die spätere Trennmauer zwischen Raum 1 und dem vergrößerten Raum 3 zerstört, Raum 27 und 32 wurden von Osten her (Raum 19) beheizt. Die Hypokausten wurden noch etwas eingetieft, so daß die älteren Böden hier den sehr massiven Hypokaustsohlen zum Opfer gefallen sind. Als Suspensur wurden Steinpfeilerchen verwendet, die oben abakusartig vergrößert sind. Darüber waren wohl Ziegelplatten verlegt, auf die der Estrich vergossen werden konnte. Von den Fußböden der beheizten Räume blieb kein Rest, da die Hypokausten in der folgenden Periode verschüttet und z. T. Schlauchheizungen eingebaut wurden. Soweit in den anderen Räumen Böden angetroffen wurden, waren das gut verlegte Steinbürsten mit Mörtelestrich. Einige dieser Böden wurden in der folgenden Periode mit einer weiteren Bürste versehen, also nochmals erhöht. Auf der dadurch geschützten Estrichfläche hat sich in Raum 25 nahe der Ostwand die Spur eines Ofens als Mörtelbänkchen erhalten. Die Funktion dieses Raumes als Küche ist daher trotz der Erhöhung des Niveaus gleichgeblieben ${ }^{23}$.

Unter dem späteren Raum 1 wurde auch ein Teil des nichtüberbauten Bereiches rund um das Gebäude erfaßt. Ein zunächst schwer verständlicher, etwa quadratischer Anbau findet so seine Erklärung als Rampe und Vorplatz zum Überwinden des Niveauunterschiedes zwischen dem unveränderten Außenniveau und dem erhöhten Niveau des Baus. Eine anzunehmende zweite Zungenmauer war nicht mehr faßbar. Eine Kieslage und anschließende Wagenspuren, die in Ost-West-Richtung vor dem Ende der Rampe vorbeiziehen und einen Wirtschaftsweg im Areal der Villa anzeigen, passen zu diesem Befund.

Als Ausgleichslage über den Schuttmassen, die für die Niveauerhöhung verwendet worden waren, konnte an vielen Stellen eine 0,10-0,20 m hohe Lage aus dunkler Erde und Sand, vielleicht mit etwas Asche vermischt, festgestellt werden. Das Fundmaterial aus dieser Lage gehört einheitlich in das 2. Jahrhundert, wobei die spätesten genauer bestimmbaren Stücke der Rest einer Reliefschüssel Drag. 37 des Cobnertus III und ein Teller Drag. 32 des Verus aus Rheinza-

${ }^{22}$ K. M. Swoboda, Römische und romanische Paläste ${ }^{3}$ (1969) 5-61.

23 S. Zabehlicky-Scheffenegger, Does form (of a vessel) correspond to function (of a room or building)? A comparison between Virunum and Bruckneudorf, Vortrag bei dem »ROCT-Workshop on Contextual Archaeology«, 12.-13. Dezember 2003 in Leuven (Belgien). 


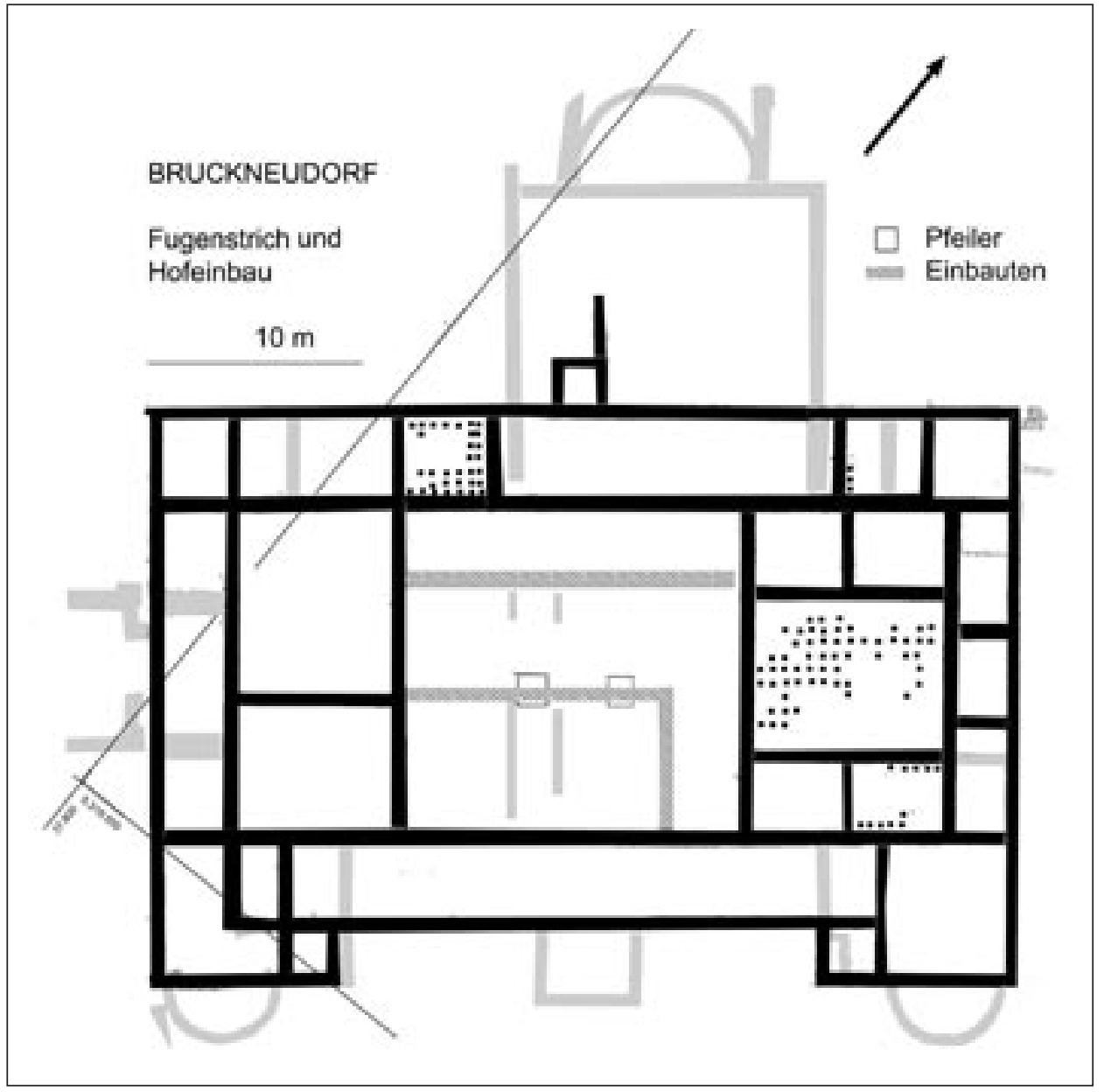

8 Plan der Fugenstrichperiode mit späteren Einbauten

bern sind. Damit ist ein terminus post quem in der zweiten Hälfte des 2. Jahrhunderts n. Chr., eher erst nach 170/80 für diese Periode gewonnen ${ }^{24}$.

Ein Einbau hat in einer späteren Phase den Hof wesentlich verändert. Schon nach der Abnahme der Mosaiken im Korridor Raum 12 zeigte sich ein massiver Pfeiler unter dem Boden. Ein zweiter wurde seither östlich davon angetroffen. Ihre Bauweise entspricht nicht der Fugenstrichperiode: die Fundamente greifen nur geringfügig in den älteren Lehmboden des Hofes ein, es wurden plattige Steine verwendet und Fugenstrich fehlt. Diese Pfeiler scheinen erst nach der Erhöhung des Niveaus errichtet worden zu sein. Wie bei anderen später eingesetzten Mauern werden sie nach unten hin breiter. Das Fundament wurde also in einer breiteren Grube aufgebaut und nicht in eine knappe Fundamentgrube eingesetzt; dieser Vorgang war wohl beim Fundamen-

${ }^{24}$ Ein stempelgleicher Teller (VERVSFECIT, Lud. V 232e) fand sich z. B. in Kumpfmühl (bald nach 171/72, wohl erst ab ca. 180): A. Faber, Das römische Auxiliarkastell und der Vicus von Regensburg-Kumpfmühl, Münchner Beiträge zur Vor- und Frühgeschichte 49 (1994) 210 und Beil. 5, 95 (mit weiteren zeitgleichen Parallelen), Cobnertus III auch noch im Keller von Sulz: A. Schaub, Markomannenkriegszeitliche Zerstörungen in Sulz am Neckar - Ein tradierter Irrtum. Bemerkungen zu reliefverzierter Terra Sigillata vom Ende des zweiten Jahrhunderts, in: H. Friesinger - J. Tejral - A. Stuppner (Hrsg.), Markomannenkriege. Ursachen und Wirkungen (1994) 439-455 (Verfüllung post 180/83), beide im Regensburger Gräberfeld: S. v. Schnurbein, Das römische Gräberfeld von Regensburg, Materialhefte zur Bayerischen Vorgeschichte A 31 (1977) Taf. 154, 32 und 195, 4 (123: »Belegungsbeginn ... nach der Mitte des 2. Jahrhunderts, eher sogar gegen dessen Ende«). Für Literaturhinweise sei I. Huld-Zetsche und $\mathrm{M}$. Thomas gedankt. 
tieren in den lockeren Schutt der Planierung notwendig ${ }^{25}$. In sehr ähnlicher Technik und gleich tief fundamentiert sind die Mauern, die zwischen den Pfeilern eingespannt sind und östlich von ihnen gegen Süden verlaufen, sowie die Mauer, die im Norden des Hofes den Raum 8 von diesem abtrennt. Allerdings ist ein etwas anderes Steinmaterial verwendet worden, es fehlen die charakteristischen Steinplatten. Stellt man diese Baumaßnahmen zusammen, dann verkleinern sie den Hof stark. Auch der Zugang von Norden her wird behindert, weil zwei Korridore (unter Raum 1 und Raum 8) gequert werden müssen. Als nunmehr gedeckte Arbeitsfläche ergibt sich der Bereich der Räume 24 und 29 einschließlich des südlichen Teiles von Raum 12. Offen bleiben die Flächen der Räume 11, des Nordteils von 12, 30 und Raum 31 als Zugang zur Porticus im Süden. Eine bessere Datierung als nach der Errichtung des Fugenstrichbaus (post ca. 170/180 n. Chr.) und vor der Periode mit Ziegeldurchschuß (nach der Mitte des 4. Jahrhunderts n. Chr.) ist derzeit nicht zu geben.

Eine Beurteilung dieses Grundrisses zeigt ihn als Weiterentwicklung der 2. Periode mit einigen Zusätzen und - in wörtlicher Bedeutung - auf einem höheren Niveau. Zunächst bleibt der zentrale Hof erhalten, im Sinne des oben Gesagten ist er kein Peristyl, da keine Stützenstellungen nachweisbar sind, die zu 'Hallen' entlang den Hofmauern gehören können. Die Südfront erhält eine Porticus und ausgeprägte rechteckige Eckrisaliten. Zur Porticus gehören wohl die wenigen Fragmente von Säulenteilen, die gefunden wurden. Es paßt in dieses Bild, daß eine Basis und ein Schaftbruchstück in der Verfüllung des Hypokaustums unter der Osthälfte von Raum 32 angetroffen wurden, wohin sie wohl gelangt sind, als man später den ganzen Raum 32 mit einer Schlauchheizung versehen hat und den Hohlraum des Hypokaustums teilweise auffüllen mußte. Mit dem Raum 27 wird ein Saal repräsentativer Größe verbunden mit der Bequemlichkeit einer Hypokaustheizung geschaffen. Auch seine axiale Stellung in bezug auf den zunächst noch nicht verbauten Hof hebt ihn heraus. In Position und Größe entspricht er gut dem Raum 20 im Hauptgebäude der Villa von Baláca (Ungarn), der auch an das Ende des 2. Jahrhunderts $\mathrm{n}$. Chr. gesetzt wird $^{26}$. In Baláca ist jedoch der Hof größer und durch seinen Peristylcharakter auch repräsentativer. Nach heutigem Wissen sind Baláca und Bruckneudorf die größten Villen der Provinz Pannonien.

Die Periode mit Ziegeldurchschuß und Mosaikböden

Das wichtigste Merkmal der Periode mit Ziegeldurchschuß und Mosaikböden ist gewiß der umfangreiche Mosaikschmuck. Er wurde in den Berichten über die früheren Grabungsperioden schon ausführlich berücksichtigt ${ }^{27}$ und in Arbeiten über Mosaiken gesondert gewürdigt ${ }^{28}$. Hier wird versucht, die einzelnen Böden in den Gesamtplan einzufügen (Abb. 9). Eine neue Gesamtbewertung des Mosaikbestandes ist geplant, kann aber nicht durch den Verfasser erfolgen, der dafür kein Spezialist ist.

Bautechnisch scheint diese Periode durch einen ein- oder mehrlagigen Ziegeldurchschuß in den Mauern bestimmt zu sein, der sich am deutlichsten bei der Westmauer von Raum 1 zeigt (Abb. 2). Daß dieser Raum als später Anbau zu verstehen war und die Nordmauer des älteren Gebäudes überbaut hat, wurde schon von B. Saria erkannt ${ }^{29}$. In der weniger ausgeprägten ein-

\footnotetext{
25 Diese Baugruben konnten im Planum nicht erkannt werden, was nicht verwundert, wenn dasselbe Schuttmaterial bald nach dem Bauvorgang wieder eingefüllt worden ist.

26 S. Palágyi, Schwerpunkte der neuen Ausgrabungen im Gutshof von Baláca, in: Balácai Közlemények III (1994 [1995]) $10-21$ bes. 13 und Abb. 4.

27 Saria (Anm. 4); Langmann (Anm. 5).

${ }^{28}$ H. Kenner, Römische Mosaiken aus Österreich, in: La mosaïque gréco-romain, Colloques Internationaux du Centre National de la Recherche Scientifique (1965) 85-94 bes. 89-90; W. Jobst, Antike Mosaikkunst in Österreich (1985) 112-119.

29 Saria (Anm. 4) 253.
} 


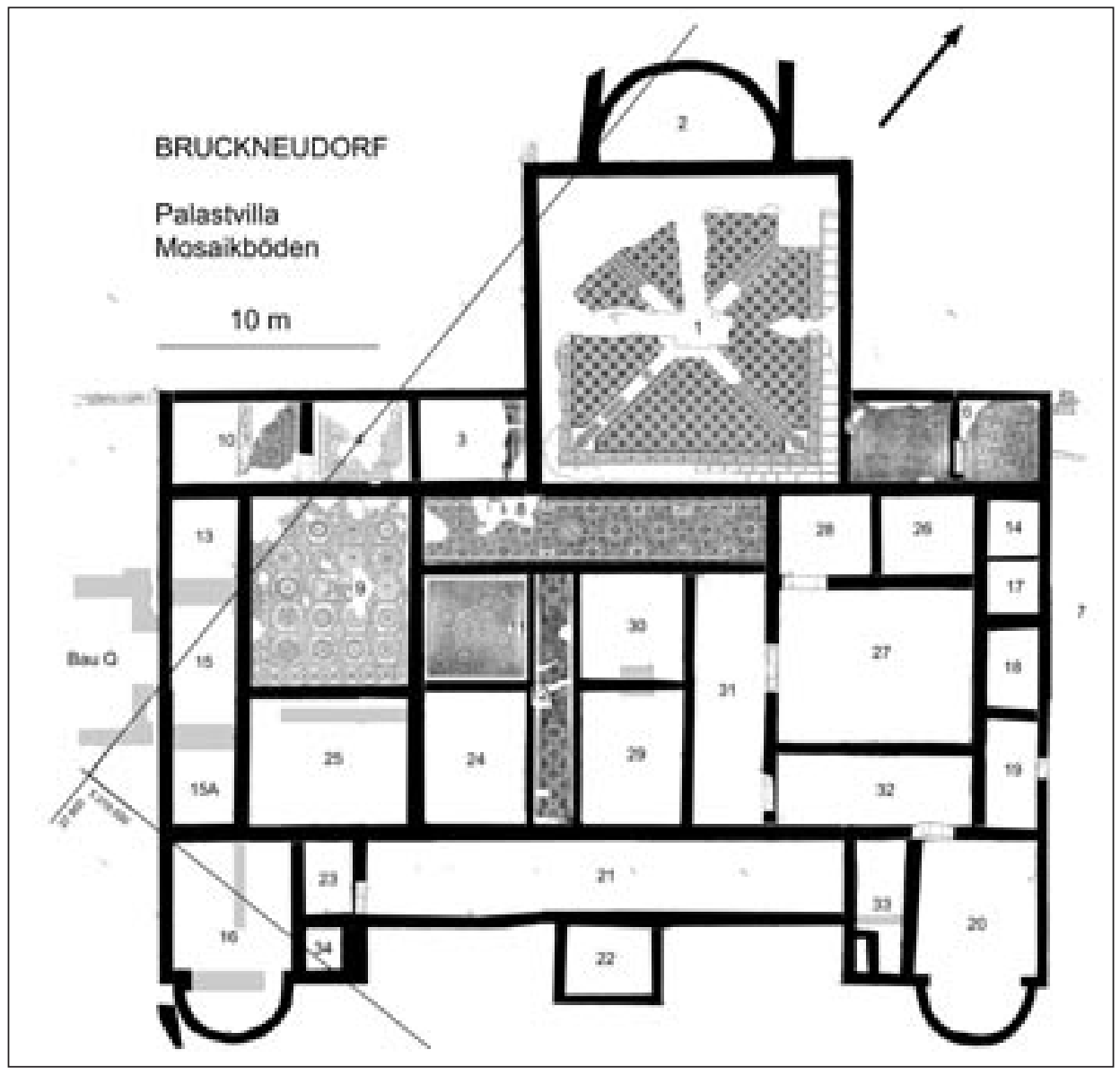

9 Plan der Periode mit Ziegeldurchschuß und Mosaikböden mit Raumnummern

scharigen Form zeigt sich Ziegeldurchschuß auch an den Apsismauern der Räume 16 und 20, wo auch die Pfeiler an den Apsisansätzen komplett aus Ziegeln errichtet sind, sowie an dem kleinen Anbau Raum 22 in der Mitte der Südfront.

Ziegeldurchschuß zeigen auch die oben im Anschluß an die Pfeiler erwähnten Mauern des Hofeinbaus; dort kann er am ehesten als Ausbesserung verstanden werden. Das Mosaik in Raum 12 überdeckt jedenfalls nicht nur den westlichen Pfeiler, sondern auch eine Mauerspur, die an der erhaltenen Oberkante des Pfeilers sichtbar ist. Schon mit der Überbauung durch die Mauer hat der Pfeiler also seine Funktion verloren. Da die Mauerspur aber auch unter dem Mosaik lag, war die Mauer jedenfalls dort, wo sie durch den Raum 12 verlief, ebenfalls kassiert worden. Westlich und östlich davon trennt sie noch die Räume 11 und 24 bzw. 29 und 30 voneinander. Sehr schmal und ungewöhnlich seicht fundamentiert sind die Längsmauern von Raum 12 und die Mauer zwischen Raum 30 und der Nordhälfte von Raum 31. Erst durch diese Baumaßnahmen entstehen die Räume 11, 12, 24, 29, 30 und 31, die jetzt den gesamten früheren Hof verbauen.

Typisch für diese Periode ist auch der Einbau von Schlauchheizungen bzw. von Hypokausten, die nur unter einem Teil des Raumes liegen; auf dem Plan der Heizkanäle dieser Periode sind die älteren Mauern nicht dargestellt (Abb. 10). In den nunmehr apsidalen Räumen 16 und 20 werden Teilflächenhypokausten eingebaut, der Saal Raum 1 erhält eine mehrfach verzweigte Schlauchheizung, sonst werden T-förmige Schlauchheizungen errichtet. In Raum 3 wird das Hypokaustum verfüllt und eine T-Heizung eingerichtet, die nun, da der Raum an den großen Saal grenzt, von Norden aus bedient werden muß. Raum 10 wird nach Osten vergrößert und erhält eine wieder von Norden zu bedienende Schlauchheizung, die den untersten Teil der älteren 


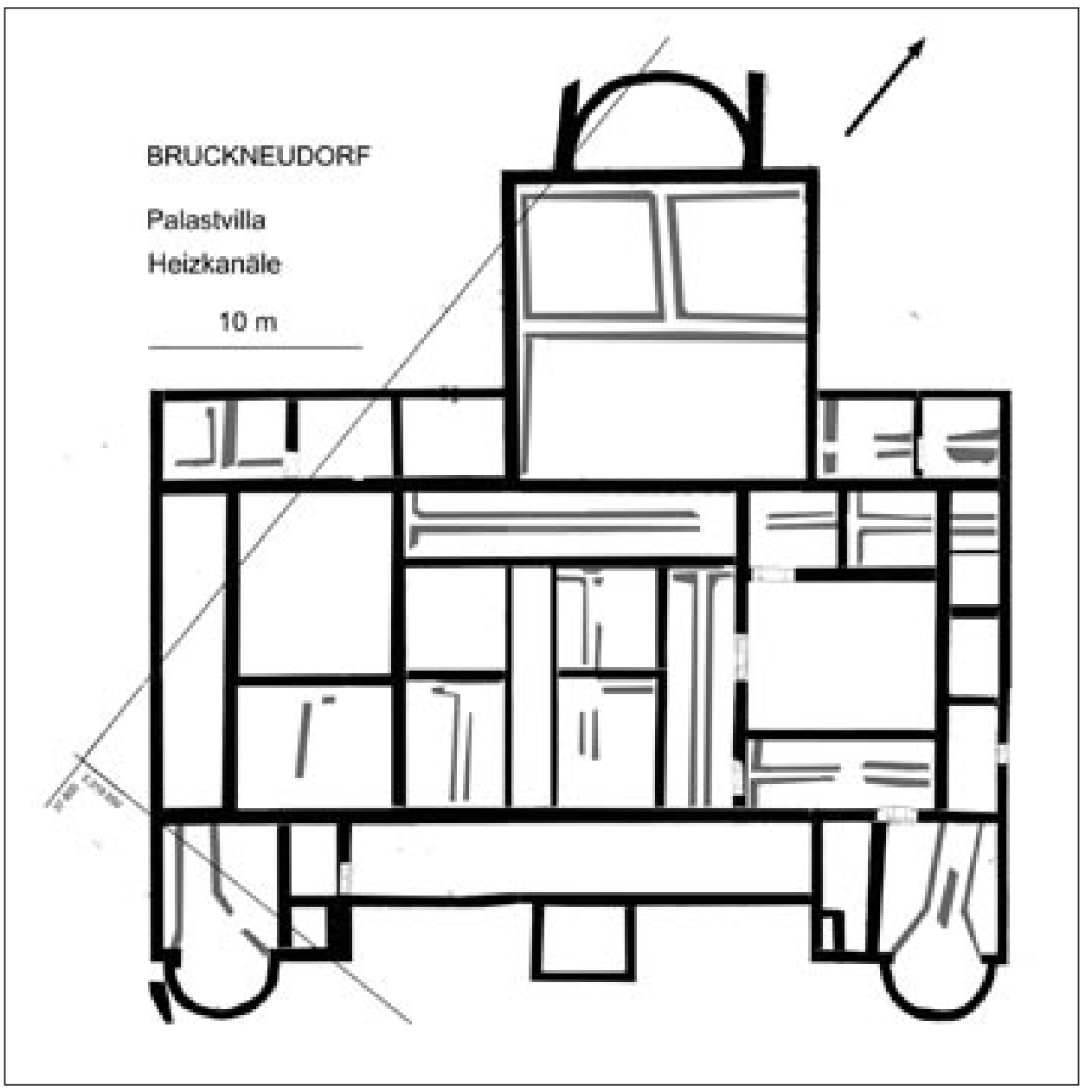

10 Plan der Periode mit Ziegeldurchschuß und Mosaikböden mit Heizkanälen

Raumtrennmauer als Kanalwange nutzt. Die Räume 5 und 6 erhalten hintereinandergeschaltete T-Heizungen. Ein ungewöhnlich lang ausgestaltetes Heizsystem, wie das vorige von Osten aus beheizt, verbindet die Räume 14, 26, 28 und 8. Während die Heizung in Raum 27 offenbar ganz aufgegeben wird, erhält der Raum 32 statt des Hypokaustums unter der Osthälfte eine T-Heizung, die gemeinsam mit dem Teilflächenhypokaustum des Raumes 20 von Raum 19 beheizt wird. Das Teilflächenhypokaustum unter Raum 16 wurde von Raum 15A aus beheizt. In den beiden jetzt apsidal abgeschlossenen Risaliten 16 und 20 sind die Hypokaustsohlen ganz außerordentlich massiv gebaut. Als bis zu 0,50 m starke 'Bürste' liegen 2-3 Steinscharen in sehr hartem Mörtel.

Heizkanäle fanden sich auch unter den Räumen 24, 25 (dort sicher zweiphasig) sowie hintereinandergeschaltet in den Räumen 29 und 30 und über die gesamte Länge von Raum 31. Dabei kommt für den Raum 25 eine Bedienung von Raum 23 her in Frage, obwohl der Befund an dieser Stelle sehr gestört war. Sehr auffallend ist der Befund in Raum 31: Es war ein Heizkanal eingebaut, doch fehlt ein Praefurnium (Abb. 11). Die Südmauer ist eine solide Fugenstrichmauer ohne Spuren einer Ausbesserung ${ }^{30}$. Es bleibt als naheliegende Interpretation, daß hier eine Heizung geplant war, die Heizkanäle errichtet und wieder abgedeckt wurden, es aber nicht mehr zum Durchbruch des Praefurniums kam. Andererseits ist in der Mauer zwischen den Räumen 12 und 24 ein Praefurnium eingebaut, doch gibt es keine zugehörigen Kanäle. Die Bauweise der

30 Die Mauer kann nicht »fast von Grund auf neu« (wie Langmann [Anm. 5] 75 meint) erbaut sein, da sie nach ihrer Bautechnik und ihrer Funktion zur Fugenstrichperiode gehört. 
Heizkanalwangen ist unterschiedlich, die meisten sind sehr leicht gebaut, gelegentlich werden Tubuli wie moderne 'Hohlblocksteine' verwendet. Zu Raum 22 führt ein Kanal unter dem Raum 21 hin, und sein Estrich zeigt den Abdruck einer Unterteilung in zwei Hälften.

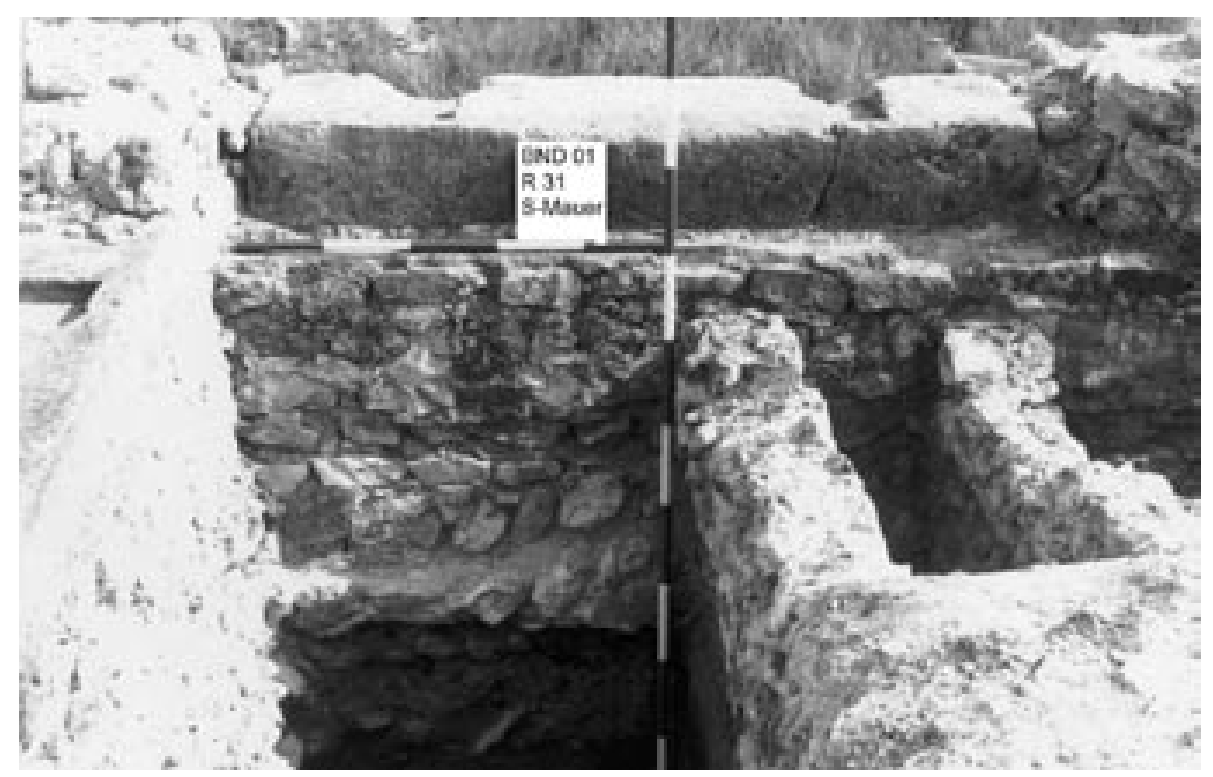

11 Heizkanal ohne Praefurnium am Südende von Raum 31

Die Ostmauer

ist durch eine überwölbte, aus Ziegeln gemauerte Öffnung durchbrochen. An der Wand wurden auch Sinterreste festgestellt.

Zur Datierung dieser Bauvorgänge hat schon G. Langmann Münzen aus aussagekräftiger Fundlage herangezogen, Prägungen von Constantin I, Constantius II und Iulian Apostata ${ }^{31}$. Auch in den Kampagnen seit 1994 sind solche Funde geglückt. Aus der Unterlage für den Boden des Raumes 32, der zur Schlauchheizung gehört, stammen Münzen von Constantinus I, Constantius II und Constans, womit jedenfalls ein Datum nach der Mitte des 4. Jahrhunderts n. Chr. gegeben ist.

Dieser Ausbau umfaßt den Anbau von Raum 1 mit der Apsis Raum 2, die apsidale Gestaltung der Eckrisaliten, den Anbau von Raum 22 an die Porticus, die Verbauung des gesamten Hofbereiches und die großzügige Ausstattung mit Mosaiken. Er schafft einen Bau, der wohl als Palastvilla angesprochen werden kann. Die gedeckte Grundfläche mißt ca. $1300 \mathrm{~m}^{2}$, davon waren annähernd $400 \mathrm{~m}^{2}$ mit Bodenmosaik geschmückt. Die Rauminterpretation für diesen Bauzustand ist schon mehrfach erfolgt ${ }^{32}$. Sie kann kurz zusammengefaßt und in einigen Punkten ergänzt werden: Raum 1 mit Apsis wird als repräsentativer Raum, gelegentlich als Audienzsaal bezeichnet. Die mosaikgeschmückten Räume im Nordostteil werden als Wohnräume des Eigentümers angesprochen, auch weil in Raum 14 eine Latrine existierte, die den 'herrschaftlichen' Bewohnern allein zur Verfügung stand; im Nebenraum 17 mündete auch eine Wasserleitung in ein Becken. Die mosaikverzierten Räume im Nordwestteil gelten als Repräsentationsräume mit offiziösem Charakter. Ob Raum 25 auch noch in dieser Phase eine Küche war, kann nicht gesagt werden. Die Räume 8, 12 und 31 sind als Gänge anzusprechen.

Die massiven Unterböden unter den Apsiden der Eckrisaliten könnten so verstanden werden, daß darüber beheizte Wasserbecken lagen. Allerdings existiert ein eigenes Bad neben dem Hauptgebäude, so daß eine solche Funktion wenig wahrscheinlich ist. Eine andere Erklärung wäre, daß für diese zweistöckigen Bauteile die Fundamentierung besonders massiv geplant wurde. In Raum 33 hat sich ein abgewinkeltes, vorwiegend aus Ziegeln errichtetes Mauerstück erhalten, das als Stiegenunterbau zu deuten ist, womit die Zweigeschossigkeit belegt wäre. Für Raum 22 wird die Funktion eines Klär- und Sammelbeckens vorgeschlagen. Nach der vollständigen Verbauung des

\footnotetext{
31 Langmann (Anm. 5) 71.

32 Saria (Anm. 4) 253 ff.; Langmann (Anm. 5) 76 ff.; Benda (Anm. 9) 40 ff.
} 
Hofes wurde die Ableitung des Dachwassers notwendig. So erklärt sich der Kanal unter Raum 21, der in Raum 22 führt. Die Innenteilung könnte zwei Beckenhälften trennen, die Wasser auffingen und kontrolliert nach Osten ableiteten.

Für das Ende dieser Phase ist eine Beobachtung wichtig, die B. Saria gelang: Er fand verschmolzenes Blei neben verkohlten Holzbalken und Dachziegeln. Das Blei ist einer Dachverblechung (Ixe) zuzuordnen, es handelt sich wohl um ein abgebranntes Dach ${ }^{33}$. Seiner Datierung an das Ende des 4. Jahrhunderts n. Chr. muß man sich aber nicht anschließen.

\section{Spätere Umbauten}

Im südöstlichen Teil des Baus wurde der beschriebene Zustand jedenfalls noch mehrfach umgebaut. Hier hat schon B. Saria unter Raum 20 eine Abfolge von drei Heizsystemen festgestell $t^{34}$. Das älteste ist das erwähnte Teilflächenhypokaustum (Abb. 12). In einem ersten Umbau wird es von einem neuen Praefurnium beheizt, das in Raum 33 liegt, von dem aus ein neuer Heizschlauch angelegt wird, der noch den alten Hohlraum versorgt. Wohl gleichzeitig erhält auch Raum 32 ein neues Praefurnium, das auch über einen neuangelegten Kanal die vorhandenen Kanäle von Raum 33 aus beheizt. Raum 19 wird also nicht mehr als Praefurnium verwendet. Es kommt aber nochmals zur Neuanlage einer Heizung in Raum 20: Ihr Praefurnium liegt wieder in Raum 33, aber nördlich neben dem genannten und höher als dieses. Es hat sich nur mehr ein Teil der Unter-

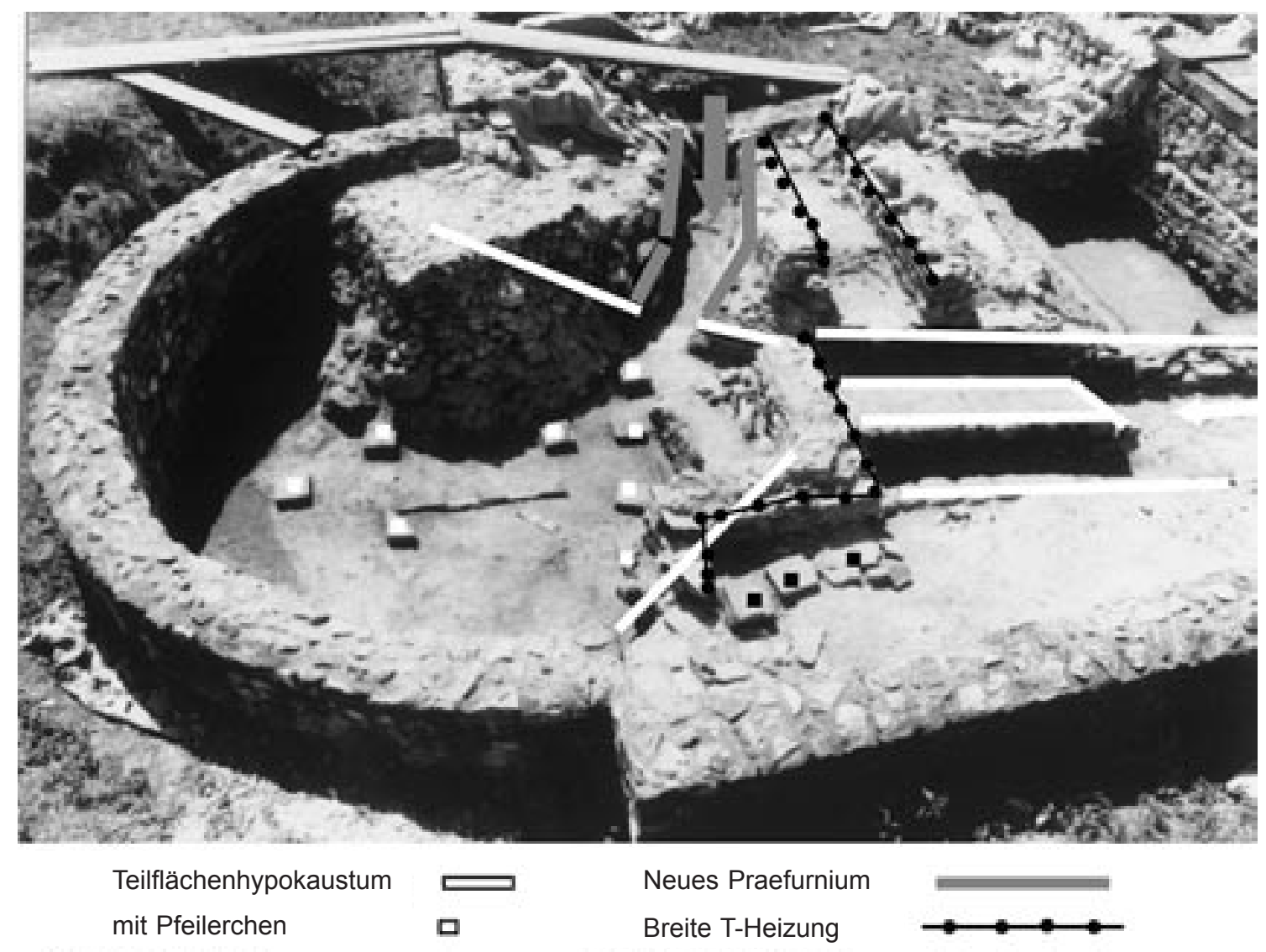

12 Drei Heizsysteme im Eckrisaliten Raum 20

33 Saria (Anm. 4) 271.

34 Ebenda $254 \mathrm{f}$. 
bodenkonstruktion dieser Heizung erhalten, der zu einem verbreiterten T-Kanal rekonstruiert werden kann.

Beginn und Dauer dieser Periode sind kaum verläßlich zu beurteilen. Die Mosaikenperiode war, da Abnutzungsspuren besonders in Raum 8 beobachtet ${ }^{35}$ und auch Mörtelausbesserungen an den Böden durchgeführt wurden, sicher einige Zeit in Verwendung.

Raum 33 hat also seit dem Einbau des neuen Kanals für das Teilflächenhypokaustum als Praefurnium gedient. Es bleibt unklar, ob er in dieser Zeit auch noch Stiegenhaus war. Eine Zerstörung oder Aufgabe des Oberstocks ist also möglich. Da sich die späten Umbauten nur im Südosten des Baus nachweisen lassen, könnte man auch an eine teilweise Zerstörung denken, die eben hier Bauteile verschont hatte, die noch adaptiert und umgebaut werden konnten. Auch die spätesten Funde stammen aus diesem Teil des großen Gebäudes, u. a. eine spätantike Lampe Iványi XXII aus der Mitte von Raum $21^{36}$. Späte Funde streuen von der Südostecke des Gebäudes bis $12 \mathrm{~m}$ nach Norden und $20 \mathrm{~m}$ nach Westen. Allerdings sind insgesamt die Schichten über den Böden schon in den früheren Grabungsperioden weitgehend entfernt worden; damit sind Schlüsse aus der Fundstreuung nicht sehr verläßlich. Als Überlegung sei angemerkt, daß die so bestimmte Fläche gut der Villa von Höflein entspricht ${ }^{37}$, die im 4. Jahrhundert und bis in den Beginn des 5. Jahrhunderts bestanden hat ${ }^{38}$. Der umschriebene Platz reicht also für ein kleineres Hauptgebäude aus, doch ist hier keine Sicherheit zu gewinnen.

\section{Die Stellung des Gutshofes im Hinterland von Carnuntum und erste Interpretationen}

Schon B. Saria und G. Langmann haben betont, daß das Bestehen eines so ungewöhnlich großen Gutshofes an dieser Stelle nur in Zusammenhang mit der Lage im Hinterland von Carnuntum verständlich ist ${ }^{39}$. Ein Versuch, die Siedlungsmuster zum Zeitpunkt der Errichtung des Legionslagers und später darzustellen, zeigte eine ausnehmend dünne Besiedlung ${ }^{40}$.

Für die Versorgung eines Standlagers war eine ausreichende landwirtschaftliche Produktion im Hinterland von Vorteil, auch wenn das römische Heer die Versorgung über weite Strecken sicherstellen konnte. Es scheint daher nach der Stationierung der Legion eine Aufsiedelung stattgefunden zu haben, in deren Verlauf auch der erste Bau an dieser Stelle, der erwähnte Holzbau, errichtet wurde. Die Lage der Villa folgt einer Regelmäßigkeit, die beispielsweise in Obergermanien ${ }^{41}$ festgestellt worden ist: Sie liegt an einer Grenze zwischen Feuchtböden und trockeneren Böden, was Getreideanbau auf den trockeneren und Viehhaltung auf den feuchteren Flächen ermöglichte.

Die auffallende Änderung der Orientierung bald nach dem zumeist flavisch datierten Limesausbau ist kein Einzelfall in dieser Region. In Carnuntum wird das Legionslager in seiner 3. Periode neu orientiert ${ }^{42}$, ebenso das Auxiliarkastell in Petronell in seiner Phase $2^{43}$. In Bruckneudorf ist dieselbe Änderung zwischen der Holzbauperiode und der Periode der 'älteren Fun-

35 G. Langmann hat diese Beobachtung im Tagebuch zum 5. Juni 1975 vermerkt, ist in den Publikationen aber nicht darauf eingegangen.

36 Vgl. auch E. Alram-Stern, Die römischen Lampen aus Carnuntum, RLÖ 35 (1989) 52-54.

37 R. Kastler, Archäologie in Höflein bei Bruck an der Leitha, CarnuntumJb 1995, 215-255 bes. 252.

38 R. Kastler, Archäologie in Höflein bei Bruck an der Leitha, CarnuntumJb 1998, 135-259 bes. 203 ff.

39 Saria (Anm. 4) 270; Langmann (Anm. 5) 113.

40 H. Zabehlicky, Gedanken zur Siedlungsstruktur im Hinterland von Carnuntum, in: N. Gudea (Hrsg.), Roman Frontier Studies, Proceedings of the XVIIth International Congress of Roman Frontier Studies (1999) 623-627.

${ }^{41}$ M. Klee, Die ländliche Besiedlung und Landwirtschaft des linksrheinischen Obergermanien, in: H. Bender - H. Wolff, Ländliche Besiedlung und Landwirtschaft in den Rhein-Donau-Provinzen des römischen Reiches, Passauer Universitätsschriften zur Archäologie 2 (1994) 199-236 bes. 202.

42 M. Kandler, Die Ausgrabungen im Legionslager Carnuntum 1968-1973, AnzWien 111, 1974, 27-40 bes. 33 f.

43 H. Stiglitz, Gedanken zur Kastellvermessung, in: dies. (Hrsg.), Das Auxiliarkastell Carnuntum 1, Forschungen 1977-1988, SoSchrÖAI 29 (1997) 136-139. 
damente' festzustellen. Diese Änderungen erfolgen im Uhrzeigersinn um $5^{\circ}$ im Auxiliarkastell ${ }^{44}$, um jeweils $7^{\circ} \mathrm{im}$ Legionslager und in Bruckneudorf. Es wird noch zu untersuchen sein, ob diese Änderung auf eine Neuvermessung im Zuge oder in Folge der Erhebung von Carnuntum zum municipium Aelium zurückgeführt werden kann.

Der Verfasser hat schon bei einer früheren Gelegenheit einen Zusammenhang der Periode der 'älteren Fundamente' mit dem schon nach seiner Auffindung viel diskutierten Grabstein des Cocceius Caupianus pr. c. B. vorgeschlagen ${ }^{45}$. Dieser princeps civitatis Boiorum ${ }^{46}$ hätte also ein Anwesen, das nicht einem durchschnittlichen einheimischen Gehöft entsprach, bewohnt. Zur einheimischen Bauweise paßt weder die reichliche Verwendung von Rutenputz noch die Anlage um einen rechteckigen Hof; beides wurde wohl aus 'römischer' Bauweise übernommen. Auch die Sprache der Inschriften, die als Spolien in spätantiken Gräbern nächst der Villa gefunden wurden, scheinen einen Einblick in den Vorgang der 'Latinisierung' oder 'Romanisierung' der keltischen Bevölkerung zu geben ${ }^{47}$.

Die Fugenstrichperiode ist durch eine sehr deutliche Erhöhung der Bodenniveaus und durch den Einbau von Hypokaustheizungen charakterisiert. Der oben angegebene terminus post quem erlaubt es, diesen Umbau in eine Reihe von Wohnbauten von Britannien bis Kleinasien zu stellen, die in den letzten Jahrzehnten des 2. Jahrhunderts n. Chr. mit Heizungen versehen wurden. Derartige Maßnahmen können als Folgen einer kurzfristigen Klimaverschlechterung verstanden werden ${ }^{48}$.

Die in ihrer Zeit nahe dem Donaulimes ungewöhnlich prächtige Ausstattung hat natürlich Anlaß gegeben, die Mosaikenperiode mit einem historisch bekannten Ereignis in Verbindung zu bringen. B. Saria hatte für diese nach damaligen stilistischen Kriterien eine Datierung um 300 n. Chr. angenommen und die Möglichkeit erwogen, daß ein Teilnehmer der 'Kaiserkonferenz von Carnuntum' 307 oder 308 hier residiert hätte ${ }^{49}$. Noch vor der Grabungsperiode ab 1976, also bevor bekannt wurde, daß die Münzfunde den zeitlichen Ansatz auf die Zeit nach 355 festlegen würden, hat A. Mócsy die Villa in einen Zusammenhang mit der schriftlich überlieferten Geschichte gebracht ${ }^{50}$. Danach wäre die Villa zum Zeitpunkt des Todes Valentinians I. im Herbst 375 Aufenthaltsort seiner Frau und seines Sohnes gewesen. Erwartungsgemäß haben auch die neueren Grabungen keinen Fund gebracht, der diese Theorie beweisen oder widerlegen könnte. Als Argument für diese Hypothese könnte aus dem Befund abgeleitet werden, daß der Umbau einerseits ein höchst repräsentatives Bauwerk mit Mosaiken geschaffen hat, andererseits aber sehr flüchtig ausgeführt wurde. Die nie in Betrieb gegangenen Heizkanäle sprechen für Planungsänderungen noch in der Ausführungsphase. Das kann mit einer Situation verbunden werden, in der die Ankunft des Hofstaates in naher Zukunft erwartet wird und während der auch widersprüchliche Angaben über die Wünsche der 'allerhöchsten Herrschaften' gemeldet werden. Der Grundriß, die Größe der gesamten Anlage und besonders die Maße des Raumes 1 weichen nicht so sehr von der Villa in $\mathrm{Konz}^{51}$ ab, die als kaiserliche Sommerresidenz gedeutet wird, als daß

44 Im Auxiliarkastell erfolgt zusätzlich eine Umorientierung um $90^{\circ}$, die mit der Funktion oder der gedachten 'Feindrichtung' zusammenhängt, aber kaum mit der römischen 'Landesvermessung'.

45 H. Zabehlicky, Fundus Cocceianus, oder »Wem gehörte die Villa von Bruckneudorf?«, in: P. Scherrer - H. Taeuber - H. Thür (Hrsg.), Steine und Wege. Festschrift D. Knibbe, SoSchrÖAI 32 (1999) 397-401.

46 Überlegungen zu der angesichts der kurzen Regierungszeit des Nerva beachtlichen Anzahl von Coccei in den Donauprovinzen sollen unter dem Titel »Coccei principes prope ripam Danuvii« demnächst in Acta Musei Napocensis erscheinen.

47 Zabehlicky (Anm. 45) 400.

${ }^{48}$ H. Zabehlicky, Kriegs- oder Klimafolgen in archäologischen Befunden?, in: Friesinger - Tejral - Stuppner (Anm. 24) 463-469 bes. 446.

49 Saria (Anm. 4) 271.

50 A. Mócsy, A párndorfi villa kérdéséhez - Zum Herrensitz von Parndorf, Dissertationes Archaeologicae (Régeszéti Dolgozatok) Ser. I, 10 (1969) 56-64; ders., Murocincta, in: V. Miroslavjevic - D. Rendić-Miočević - M. Suić (Hrsg.), Adriatica Praehistorica et Antiqua, Miscellanea Gregorio Novak dicata (1970) 583-586.

51 A. Neyses, Die spätrömische Kaiservilla zu Konz (1987) Plan S. 14. 
diese Interpretation auszuschließen ist. Auch die Interpretation der erhaltenen figürlichen Mosaiken kann in einen erstaunlich engen Zusammenhang mit dieser Theorie gebracht werden: bei der Deutung der weiblichen Gottheit im Medaillon des Raumes 11 schwankte B. Saria zwischen Ceres und Diana, G. Langmann hat sich sehr eindeutig auf Diana festgelegt ${ }^{52}$. Der Verfasser hat sich mit einigen Argumenten für Ceres ausgesprochen ${ }^{53}$ und einen Zusammenhang mit dem Bericht des Ammianus Marcellinus hergestellt: arma parabat et alimenta ${ }^{54}$. Die militärische Rüstung und die Vorbereitung der Versorgung konnte wohl kaum besser illustriert werden als durch Bellerophon und Ceres.

Gegen die Hypothese können aber die Münzfunde unter den Böden angeführt werden: és waren Prägungen der Constantinsöhne, die einen terminus post quem von $355 \mathrm{n}$. Chr. nahelegen. Da der Geldumlauf der valentinianischen Zeit von jüngeren Stücken dominiert wird ${ }^{55}$, könnte man das Datum dieser intensiven Bautätigkeit näher an den terminus post quem heranrücken. Auch im nahegelegenen Carnuntum nimmt der Münzzustrom in valentinianischer Zeit deutlich $\mathrm{zu}^{56}$. Die massive Bautätigkeit am Limes, die diese große Geldmenge in die Region gebracht hat, war ja der Anlaß für die quadische Unruhe und in der Folge für die Anwesenheit des Kaisers ${ }^{57}$. Fiele die Bautätigkeit in das Jahr 375, wären Prägungen der valentinianischen Dynastie zu erwarten. Damit ist eine intensive Bautätigkeit im Jahr 375 keineswegs auszuschließen, sie ist aber weniger wahrscheinlich.

Es kann eine weitere Erklärung überlegt werden, die zu dieser chronologischen Einordnung besser paßt. Für Carnuntum hat M. Kandler eine Erdbebenzerstörung um die Mitte des 4. Jahrhunderts n. Chr. angenommen ${ }^{58}$. Auch in Bruckneudorf unterstützt die Tatsache, daß das nahe der Villa gelegene Gräberfeld zu jenen gehört, die einen ungewöhnlich großen Anteil Steinkistengräber mit Spolien haben, die Vermutung, daß dieses Erdbeben die älteren Gräber zerstört hat ${ }^{59}$. So wäre eine denkbare Interpretation, daß hier eine vielleicht weniger beschädigte Villa als offizielle Residenz, eventuell als Statthalterpalast, ausgebaut wurde, nachdem der Amtssitz in Carnuntum zerstört worden war. Ein vergleichbarer Palastbau ist aus der Kyrenaika, allerdings erst aus dem frühen 6. Jahrhundert bekannt. In Apollonia entsteht ein 'Palast des Dux'. Während die Gesamtanlage dort größer ist, hat die Audienzhalle einschließlich der Apsis ein Maß von ungefähr $20 \times 12 \mathrm{~m}^{60}$, sie kann also mit dem Bruckneudorfer Befund verglichen werden.

So wie die Bemerkungen zum Befund sind auch die Versuche, das Hauptgebäude der Villa in seiner langen Geschichte zu deuten und in sein räumliches und zeitliches Umfeld einzuordnen, vorläufig, und es wird notwendig sein, vieles zu ergänzen und zu vertiefen. Trotzdem sollten

52 Saria (Anm. 3:1951) 57 f.; Langmann (Anm. 5) 106 f.; E. Simon hat das Mosaik auch zu den Darstellungen der Diana gezählt: LIMC II 1 (1984) 817 s. v. Artemis/Diana Nr. 121. Sie hat gesprächsweise im November 2000 vorgeschlagen, von einem numen mixtum zu sprechen.

53 H. Zabehlicky, Das Hinterland von Carnuntum und die Villa von Bruckneudorf, Anodos 1, 2002, 227-231 bes. $229 \mathrm{f}$.

54 Amm. 30, 5, 11.

55 Über den valentinianischen Münzumlauf vgl. z. B. J. Gorecki, Studien zur Sitte der Münzbeigabe in römerzeitlichen Körpergräbern zwischen Rhein, Mosel und Somme, Bericht der Römisch-Germanischen Kommission 56, 1975 , $179-467$ bes. 306.

56 Der Münzzustrom kann nach W. Hahn, Carnuntum, FMRÖ III 1 (1976) abgeschätzt werden. Nur die Siedlungsfunde werden berücksichtigt: Für die Prägungen von Constantius II nach der Reform bis Julianus III (348-363) aus 16 Jahren 710 Stück, also 44 Stück pro Jahr. Für Valentinianus I bis Gratian (364-375) aus 12 Jahren 820 Stück, also 68 Stück pro Jahr. Mit dieser Rechnung soll nur eine grobe Abschätzung des Münzzustromes gezeigt werden, die aber doch deutlich ausfällt. Ich danke G. E. Thüry für Diskussionen zu dieser Frage und auch für den Hinweis, daß diese Münzreform jetzt in das Jahr 348 gesetzt wird.

57 Amm. 30, 5, 1-3.

58 M. Kandler, Eine Erdbebenkatastrophe in Carnuntum, ActaArchHung 41, 1989, 313-336 bes. 333.

59 H. Zabehlicky, Zur Spolienverwendung in spätantiken Gräbern des österreichischen Donauraumes, in: Lebendige Altertumswissenschaft. Festschrift H. Vetters (1985) 279-285. Die anderen dort gegebenen Erklärungsmöglichkeiten erscheinen mir heute weniger wahrscheinlich.

60 R. G. Goodchild, Kyrene und Apollonia (1971) 185 f. Plan 2. 
diese Gedanken zur Diskussion gestellt werden. Das in seiner Größe und Ausstattung ungewöhnliche Bauwerk verdient sicher noch weitere Beschäftigung ${ }^{61}$.

\section{Präsentation des Denkmals}

Wie erwähnt, war die Planung eines Schutzbaus Anlaß für die Wiederaufnahme der Grabungsarbeiten im Jahr 1994. Mit dem Abschluß dieser Arbeiten, soweit sie das Innere des Hauptgebäudes betreffen, gibt es nun keinen Grund mehr, dieses außerordentliche Denkmal nicht so zu präsentieren, daß auch eine interessierte Öffentlichkeit die Ergebnisse nachvollziehen kann. Deshalb blieb auch der Trägerverein der damaligen Initiative bestehen, der durch den Bürgermeister Franz Schmitzhofer als Obmann in der Gemeinde Bruckneudorf gut verankert ist. Er wurde in $»$ Verein zur Erhaltung und touristischen Verwertung der $>$ Römischen Palastanlage Bruckneudorf « umbenannt und hat auch in den vergangenen 10 Jahren verschiedene Aktivitäten gesetzt. Zu erwähnen ist zunächst die Herausgabe eines Kalenders für das Jahr 1996, in dem mit Unterstützung der Fa. Römerquelle die Villa und ihr Mosaikschmuck zusammen mit anderen Funden der Umgebung vorgeführt wurden. Dazu kam die Organisation eines 'Führungsbetriebes' an dem in Ausgrabung stehenden Objekt, besonders intensiv im Jahr 1996, als eine Zusammenarbeit mit der damaligen burgenländischen Landesausstellung »Reitervölker aus dem Osten. Hunnen und Awaren « auf Schloß Halbturn möglich war. Durch Gemeinde und Tourismusverband wurde in diesem Jahr auch ein 'Radfahrerunterstand' errichtet und mit einer Informationstafel versehen; die Anbindung an einen Radwanderweg läßt hier trotz der Lage abseits moderner Ortschaften eine touristische Nutzung zu. Mit der logistischen Unterstützung der Gemeinde Bruckneudorf konnten auch in den folgenden Jahren auf Wunsch Führungen angeboten werden ${ }^{62}$. Durch den Verein und unter der Mithilfe lokaler Sponsoren wurden nun bereits drei Schautafeln errichtet, um die Ruine vor Ort zu erläutern. Der Verein mußte auch als Vermittler für private Sponsorgelder fungieren, da das ÖAI für die letzten beiden Grabungsjahre leider keine finanzielle Deckung mehr zur Verfügung stellen konnte.

Der Verein hat aber auch die Basis geboten, ein neues Projekt zur Präsentation der Villa vorzubereiten und auszuarbeiten, über das kurz berichtet werden soll. Neben der ungewöhnlichen Größe ist es die Mosaikausstattung, die den Bau gegenüber anderen Ruinen hervorhebt. Verständlicherweise war der intensive Wunsch vorhanden, diese an Ort und Stelle zu zeigen. Dem stehen aber kaum zu überwindende Hindernisse gegenüber: Die Mosaiken wurden nach ihrer Abnahme im Burgenländischen Landesmuseum verlegt, wo eine Verwahrung in wohlkontrollierten klimatischen Bedingungen bezüglich Temperatur und Feuchtigkeit möglich ist. Auch in einem gut gebauten Schutzbau könnte dies nicht in gleicher Qualität gewährleistet werden. Die Lage abseits einer größeren Siedlung birgt zudem die Gefahr von Kunstraub und Vandalismus, vor der auch eine moderne Sicherheitstechnik nicht hinlänglich Schutz bieten kann. Eine Verlegung von Kopien ist hingegen wegen der ebenfalls erheblichen Kosten kaum durchzuführen, wobei auch für Kopien die Überlegungen zu den Verlegebedingungen und der Diebstahlsgefahr gelten, wenn auch in geringerem Ausmaß. Es wurde also ein diesen Gegebenheiten angepaßtes Konzept erarbeitet, das den Zustand der letzten Bauperiode darstellt. Geplant ist eine Überhöhung des antiken Mauerbestandes, soweit er noch vorhanden ist, bzw. der Neuaufbau der fehlenden Mauerstücke. Die Niveaus der Mosaiken und der anderen Böden der Ziegelduchschußperiode sollen durch eine Pflasterung angegeben werden, die wenigstens die hauptsächlichen Rapporte der Mosaikböden andeuten soll. Ergänzend sind weitere Schautafeln geplant, die die Mosaiken zeigen und selbstverständlich auf die Originale im Burgenländischen Landesmuseum verweisen.

${ }^{61}$ Der Bau hat in seiner letzten und größten Periode ein Ausmaß von $45 \times 42 \mathrm{~m}$ und ist durch seine Mosaikausstattung von $400 \mathrm{~m}^{2}$ im pannonischen Raum und darüber hinaus als außerordentliches Denkmal bekannt. Es sei ein Vergleich mit einem mediterranen Beispiel erlaubt: Das Hanghaus 2 in Ephesos mißt $47 \times 75 \mathrm{~m}$.

${ }^{62}$ Dies war auch durch das Engagement von Frau B. Petznek/Bruck a. d. Leitha möglich. 
Erläuterungen und graphische Rekonstruktionen sollen ebenfalls Inhalt dieser Tafeln sein.

Diese Vorschläge und die aus der Grabung gewonnenen Unterlagen wurden in eine Form gebracht, die eine Kostenschätzung gestattete ${ }^{63}$. Auf dieser Grundlage wurde durch den Verein an das Land Burgenland ein Antrag zur Förderung mit Mitteln der Europäischen Union eingereicht, der inzwischen bewilligt ist. Die Detailplanung ist seit dem Frühjahr 2004 im Gang. Als Beispiel für die Planung sei ein Blick in den Raum 4 vorgestellt (Abb. 13): Die z. T. bereits 1984/85 in An-

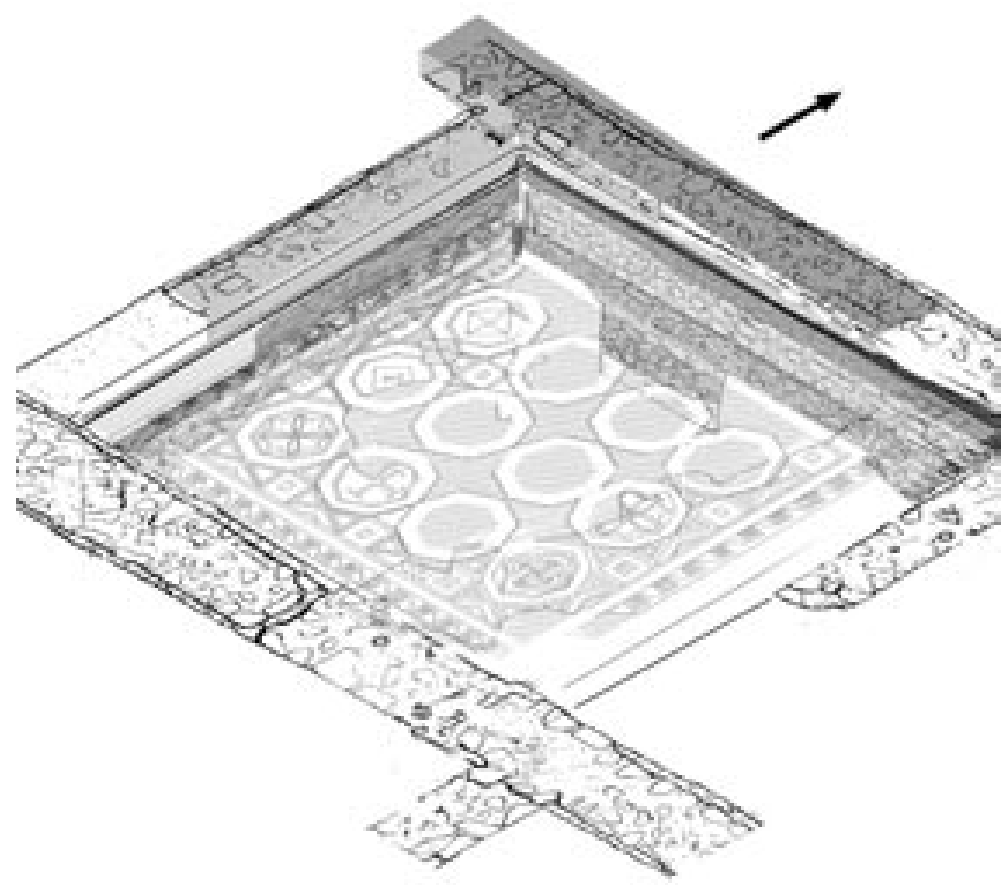

13 Mosaik und Pflasterungsvorschlag für Raum 4 (Aufnahme des Mosaiks: E. R. Stain und I. Sekal, Zeichnung E. Grubits) sätzen aufgebauten Mauern sollen noch soweit überbaut werden, daß die Böden in ihrem ursprünglichen Niveau verlegt werden können. In die zeichnerische Aufnahme des Mosaiks wurde an den Fehlstellen die vorgeschlagene Andeutung des Rapports eingetragen. Mit dieser Form der Präsentation soll der antike Bestand überdeckt und geschützt bleiben sowie der Aufwand für die permanente Pflege des Platzes möglichst gering gehalten werden. Eine Führung kann dem Besucher nur bei vorheriger Anmeldung und gegen Kostenersatz angeboten werden. Dem nichtbegleiteten Besucher, der auch durch Hinweise auf dem erwähnten Radwanderweg auf die Villa aufmerksam gemacht wird, soll mit Hilfe der Tafeln und Gestaltung der Böden zusätzliche Information gegeben werden.

\section{Dr. Heinrich Zabehlicky}

Österreichisches Archäologisches Institut, Franz Klein-Gasse 1, A-1190 Wien

E-Mail: heinrich.zabehlicky@oeai.at

Abbildungsnachweis: Alle Abb. C ÖAI Wien.

${ }^{63}$ Über Vermittlung der Gemeinde hat die Fa. Konstruktiv Bauplanungsgesellschaft/Mödling diese Aufgabe übernommen. 
